\title{
Approximate electromagnetic cloaking of spherical bodies using nonlinear transformation with improved total scattering
}

\author{
Hany M. Zamel ${ }^{1, ~ *}$, Essam El Diwany ${ }^{1}$, Hadia El Hennawy ${ }^{2}$ \\ ${ }^{1}$ Electronics Research Institute - Microwave Engineering Dept.,Egypt \\ ${ }^{2}$ Faculty of Engineering - Ain Shams University, Egypt
}

Email address:

res_ass@yahoo.com(H. M. Zamel)

\section{To cite this article:}

Hany M. Zamel, Essam El Diwany, Hadia El Hennawy. Approximate Electromagnetic Cloaking of Spherical Bodies Using Nonlinear Transformation with Improved Total Scattering. American Journal of Electromagnetics and Applications. Vol. 1, No. 2, 2013, pp. 16-29. doi: 10.11648/j.ajea.20130102.11

\begin{abstract}
Cloaking refers to hiding a body from detection by surrounding it with a coating consisting of an unusual anisotropic nonhomogeneous material. The permittivity and permeability of such a cloak are determined by the coordinate transformation of compressing a hidden body into a point or a line. In this work, the scattering properties of cloaked spherical bodies (conducting and dielectric) are investigated using a combination of approximate cloaking, where the conducting sphere is transformed into a small sphere rather than to a point, and using two types of nonlinear transformations; concave-up and concave-down. The radially-dependent spherical cloaking shell is approximately discretized into many homogeneous anisotropic layers, provided that the thickness of each layer is much less than the wavelength, and this discretization raises the level of scattering as the number of layers decreases. Each anisotropic layer can be replaced by a pair of equivalent isotropic sub-layers, where the effective medium approximation is used to find the parameters of these two equivalent sub-layers. The effect of nonlinearity in the coordinate transformation on the scattering performance is studied. The back-scattering normalized radar cross section, the scattering pattern are studied and the total scattering cross section against the frequency for different number of layers and transformed radius.
\end{abstract}

Keywords: Approximate Cloaking, Scattering by Spherical (Conducting, Dielectric) Bodies, Nonlinear Transformation

\section{Introduction}

Recently, the concept of electromagnetic cloaking has drawn considerable attention concerning theoretical, numerical and experimental aspects [1] - [8]. One approach to achieve electromagnetic cloaking is to deflect the rays that would have struck the object, guide them around the object, and return them to their original trajectory, thus no waves are scattered fromthe body [1]. In the coordinate transformation method for cloaking, the body to be hidden is transformed virtually into a point (3D or spherical configuration) or a line (2D or cylindrical configuration), and this transformation leads to the profile of $\varepsilon, \mu$ in the cloaking coating. Some components of the electrical parameters of the cloaking material $(\varepsilon, \mu)$ are required to have infinite or zero value at the boundary of the hidden object. Thisrequires the use of metamaterials which can produce such values, however, they are narrow band since they rely onusing array of resonant elements (as split ring resonators) [9] - [12].Approximate cloaking can be achieved by transforming the hidden body virtually into a small object rather than a point or a line as shown in Fig. 1, which eliminates the zero (point transformed) or infinite (line transformed) values of the electrical parameters [13], [14]. This, however, leads to some scattering, since the hidden body is virtually transformed into a small object rather than a point or a line, and the scattering decreases as the transformed sphere radius is smaller.
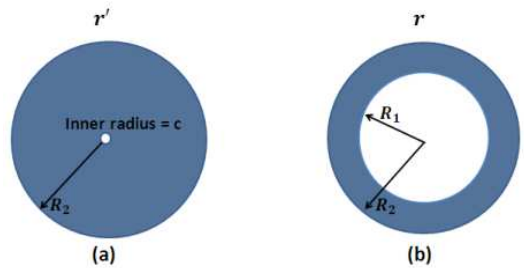

(b)

Fig. 1. (a) Virtual domain, (b) Actual Domain 
The nonlinear-transformation-based cloaks [15], [16] cannot be solved by the analytic method proposed for classic spherical cloak [17], [18], [19]. Therefore, a discretized non-ideal model has to be implemented in which each layer is homogeneously anisotropic, provided that the thickness of each layer is much less than the wavelength, and this discretization decreases the level of scattering as the number of layers increases. Each anisotropic layer can be replaced by a pair of equivalent isotropic sub-layers, where the effective medium approximation is used to find the parameters of these two equivalent sub-layers [20]. Near the boundary of the hidden sphere, the values of the radial components of $\varepsilon, \mu$ of the cloaking material are nearly zero. This makes the values of $\varepsilon, \mu$ in one of the pair of isotropic layers to be very small, and can be implemented using metamaterials [11], [21], [22]. Two approaches are possible in choosing the materialproperties in the pair of sub-layers. The first is to take the smaller values of $\varepsilon$, together with the smaller value of $\mu$ in one sub-layer, and the opposite combination in the other sub-layer [20], whereas the other possibility is to take the smaller value of $\varepsilon$ together with the larger value of $\mu$ in one sub-layer, and the opposite combination in the other sub-layer [23]. The first approach leads to improved back scattering with high forward and total scattering. The second approach leads to great reduction in forward and total scattering, while the back scattering is low enough. In this paper, we study the scattering performance of cloaked conducting and dielectric spheres using the second approach with approximate cloaking and nonlinear transformations.

\section{The Nonlinear Transformations}

Pendry's spherical cloak [1] uses a linear transformation between the radius $r$ in the virtual domain and the physical radius $r$, Fig. 1. In this section, we consider the two classes of nonlinear-transformation; the concave-up and concave-down transformations [15], [16], modified for approximate cloaking. In the concave-down and concave-up transformation, the transformation is sharper near the inner or the outer radius of the cloak, respectively. The resulting permittivity and permeability of the approximate nonlinear spherical cloak are given.

\subsection{Concave-Down Nonlinear Transformation}

The concave-down nonlinear function $f(r)$ to transform from the physical space $r$ to the virual space $r^{\prime}$ is given by:

$$
f(r)=r^{\prime}=\frac{R_{2}^{x}\left(R_{2}-c\right)}{R_{2}^{x}-R_{1}^{x}}\left[1-\left(\frac{R_{1}}{r}\right)^{x}\right]+c
$$

which obviously satisfies the requirements at the inner and outer boundaries (when $\mathrm{r}=R_{1}, r^{\prime}=\mathrm{c}$, and when $\mathrm{r}=R_{2}$, $r^{\prime}=R_{2}$ ), Fig.1. The value of " $\mathrm{x}$ " is a factor to control the nonlinearity degree in the transformation, which can be arbitrary from 0 to $\infty$.
Fig. 2 shows typical concave-down nonlinear transformation function $\mathrm{r}^{\prime}$ for different values of $\mathrm{x}$ with $\mathrm{R}_{2}=2 \mathrm{R}_{1}$. When $\mathrm{x}<0.1$, all mapping curves are overlapping (i.e. they effectively lead to the same performance). When $\mathrm{x}$ becomes large, the transformation function changessharply near the inner radius $r=R_{1}$. All curves belonging to the concave-downtransformation functions in Eq. (1) have negative second derivative with respect to the physical space radius $r$.

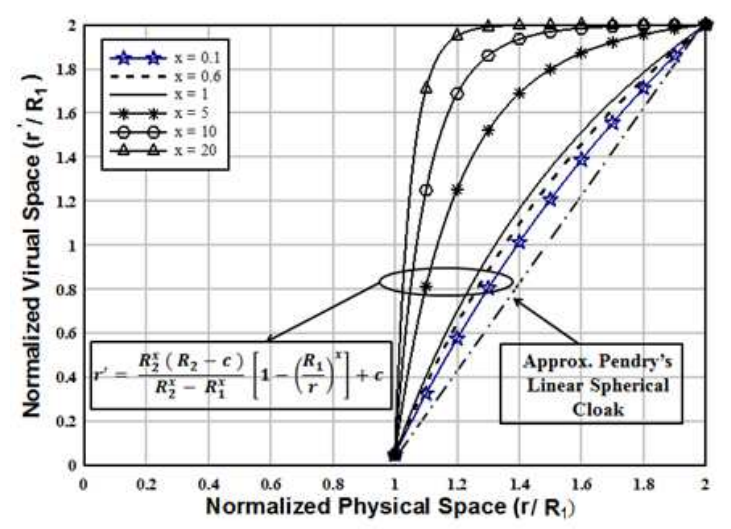

Fig. 2. The concave-down nonlinear transformation function $r^{\prime}, R_{2}=$ $2 R_{1}$ and $c=R_{1} / 20$

The radial and transverse permittivity and permeability of the spherical cloak, depending on $r$, are given as [24]:

$$
\frac{\varepsilon_{\mathrm{r}}}{\varepsilon_{0}}=\frac{\mu_{\mathrm{r}}}{\mu_{0}}=\frac{\mathrm{f}(\mathrm{r})^{2}}{\mathrm{r}^{2} \mathrm{f}^{\prime}(\mathrm{r})}, \frac{\varepsilon_{\theta}}{\varepsilon_{0}}=\frac{\mu_{\theta}}{\mu_{0}}=\frac{\varepsilon_{\emptyset}}{\varepsilon_{0}}=\frac{\mu_{\varnothing}}{\mu_{0}}=\mathrm{f}^{\prime}(\mathrm{r})
$$

Thus, the permittivity and permeability of the approximate spherical cloak in the physical space become:

$$
\begin{aligned}
& \frac{\varepsilon_{\mathrm{r}}}{\varepsilon_{0}}=\frac{\left[\mathrm{R}_{2}^{\mathrm{X}}\left(\mathrm{R}_{2}-\mathrm{c}\right)\left(\mathrm{r}^{\mathrm{X}}-\mathrm{R}_{1}^{\mathrm{X}}\right)+\mathrm{cr}^{\mathrm{X}}\left(\mathrm{R}_{2}^{\mathrm{X}}-\mathrm{R}_{1}^{\mathrm{X}}\right)\right]^{2}}{\mathrm{xR}_{1}^{\mathrm{X}} \mathrm{R}_{2}^{\mathrm{X}} \mathrm{r}^{\mathrm{X}+1}\left(\mathrm{R}_{2}-\mathrm{c}\right)\left(\mathrm{R}_{2}^{\mathrm{X}}-\mathrm{R}_{1}^{\mathrm{X}}\right)} \\
& \frac{\varepsilon_{\theta}}{\varepsilon_{0}}=\frac{\mathrm{xR}_{1}^{\mathrm{X}} \mathrm{R}_{2}^{\mathrm{X}}\left(\mathrm{R}_{2}-\mathrm{c}\right)}{\mathrm{r}^{\mathrm{X}+1}\left(\mathrm{R}_{2}^{\mathrm{X}}-\mathrm{R}_{1}^{\mathrm{X}}\right)}
\end{aligned}
$$

For modeling scattering by discretized cloak layers, it is possible to use equal thickness of the layers either in the physical space or in the virtual space. In order to avoid sharp variations of the parameters $\varepsilon, \mu$ in the inner layers, Fig. 2, the discretized layers are taken to be of equal thickness in the virtual space [15], [16] which leads to finer segmentation in the physical space,where $f(r)$ turns to be steeper. The virtual space $r^{\prime}$ is discretized into $2 \mathrm{M}$ layers with identical thickness, thus

$$
r_{i}^{\prime}=\frac{\mathrm{i}\left(\mathrm{R}_{2}-\mathrm{c}\right)}{\mathrm{M}}+\mathrm{c}, \mathrm{i}=1,2, \ldots, 2 \mathrm{M}
$$

The corresponding radii in the physical space $r$ become:

$$
\mathrm{r}_{\mathrm{i}}(\mathrm{x})=\mathrm{R}_{1} \cdot\left[1-\frac{\mathrm{R}_{2}^{\mathrm{x}}-\mathrm{R}_{1}^{\mathrm{x}}}{\mathrm{R}_{2}^{\mathrm{x}}} \frac{r_{i}^{\prime}-\mathrm{c}}{\mathrm{R}_{2}-\mathrm{c}}\right]^{\frac{-1}{\mathrm{x}}}, \mathrm{i}=1,2, . ., 2 \mathrm{M}
$$

\subsection{Concave-Up Nonlinear Transformation}

The concave-up nonlinear transformation function is 
given by:

$$
f(r)=r^{\prime}=\frac{R_{1}^{X}\left(R_{2}-c\right)}{R_{2}^{X}-R_{1}^{X}}\left[\left(\frac{r}{R_{1}}\right)^{x}-1\right]+c
$$

Fig. 3 shows typical concave-up nonlinear transformation function $\mathrm{r}^{\prime}$ for different values of $\mathrm{x}$ with $R_{2}=2 R_{1}$. When $x=1$, it is exactly Pendry's linear spherical cloak. When $\mathrm{x}$ gets extremely large, the mapping curve will become sharp near the outer radius $=R_{2}$. All curves belonging to the concave-up transformation functions in Eq. (7) have positive second derivative with respect to the physical space radius $r$.

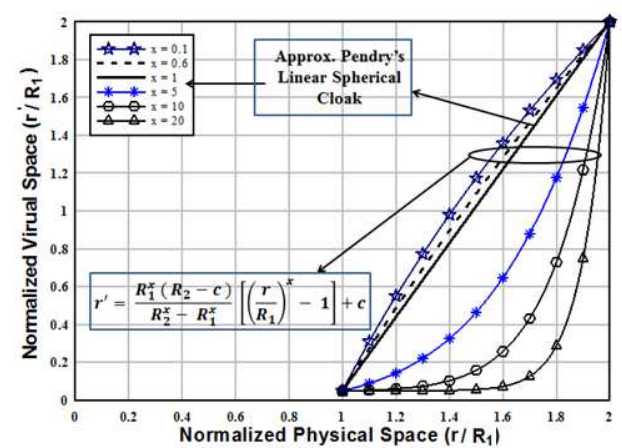

Fig. 3. The concave-up nonlinear transformation function $r^{\prime}, R_{2}=2 R_{1}$ and $c=R_{1} / 20$

In the same way, we can obtain the permittivity and permeability of the approximate spherical cloak in the physical space as:

$$
\begin{aligned}
& \frac{\varepsilon_{r}}{\varepsilon_{0}}=\frac{\left[\left(R_{2}-c\right)\left(r^{X}-R_{1}^{X}\right)+c\left(R_{2}^{X}-R_{1}^{X}\right)\right]^{2}}{x^{x+1}\left(R_{2}-c\right)\left(R_{2}^{X}-R_{1}^{X}\right)} \\
& \frac{\varepsilon_{\theta}}{\varepsilon_{0}}=\frac{x^{X-1}\left(R_{2}-c\right)}{\left(R_{2}^{X}-R_{1}^{X}\right)}
\end{aligned}
$$

As in concave-down transformation, the virtual space is discretized into equal thickness layers, Eqn. 5, the corresponding radii in the physical space $r$ turn out to be:

$$
r_{i}(x)=R_{1} \cdot\left[\frac{R_{2}^{\mathrm{x}}-R_{1}^{\mathrm{x}}}{\mathrm{R}_{1}^{\mathrm{x}}} \frac{r_{i}^{\prime}-\mathrm{c}}{\mathrm{R}_{2}-\mathrm{c}}+1\right]^{\frac{1}{\mathrm{x}}}, \mathrm{i}=1,2, . ., 2 \mathrm{M}
$$

\section{Spherical Nonlinear Cloak by Layered Structure of Homogeneous Isotropic Materials}

The fields in the cloak layer can be obtained by solving Maxwell equations using the magnetic and electric vector potentials [17], [18], [19]. The equation for the radial component of the field potentials becomes:

$$
\frac{\partial^{2} \mathrm{H}(\mathrm{r})}{\partial \mathrm{r}^{2}}+\left[\mathrm{K}_{\mathrm{t}}^{2}-\mathrm{AR} \frac{\mathrm{n}(\mathrm{n}+1)}{\mathrm{r}^{2}}\right] \mathrm{H}(\mathrm{r})=0
$$

where $\mathrm{K}_{\mathrm{t}}^{2}=\omega^{2} \mu_{\mathrm{t}} \varepsilon_{\mathrm{t}}$, (AR) denotes the anisotropy ratio of the cloak, for TM wave, (AR) $=\varepsilon_{\mathrm{t}} / \varepsilon_{\mathrm{r}}$, and for TE wave, a similar equation is obtained with $(\mathrm{AR})=\mu_{\mathrm{t}} / \mu_{\mathrm{r}}$.
For $\mu$ and $\varepsilon$ of perfect linear spherical cloaks [17]-[19],AR $=r^{2} /\left(r-R_{1}\right)^{2}$, Eq. (10) is reduced to:

$$
\frac{\partial^{2} H(r)}{\partial r^{2}}+\left[K_{t}^{2}-\frac{n(n+1)}{\left(r-R_{1}\right)^{2}}\right] H(r)=0
$$

Thus, the radial component $H(r)$ can be solved in a way similar to that for isotropic materials, except for the changein the argument of resultant Bessel/Hankel functions. However, given a set of $\mu$ and $\varepsilon$ derived from a certain transform, the anisotropy ratio may not be $r^{2} /\left(r-R_{1}\right)^{2}$ anymore, and then the radial component cannot be solved explicitly in the same way. In this situation, the original inhomogeneous anisotropic cloaking materials can be approximated by the limit of many (M) thin, concentric, homogeneous, anisotropic coating layers [15], [20]. Each anisotropic layer can be replaced by a pair of equivalent homogeneous isotropic sub-layers $\mathrm{A}$ and $\mathrm{B}$ with different thickness, where the effective medium approximation is used to find the parameters of these two equivalent sub-layers as shown in Fig. 4.

The layer thicknesses $\left(d_{A}, d_{B}\right)$ of these shells are much less than the wavelength $\lambda$ and when $d \ll \lambda$, the permittivity and the permeability relationship between the anisotropic material $\left(\varepsilon_{r}, \varepsilon_{\theta}\right)$ or $\left(\mu_{r}, \mu_{\theta}\right)$ and the two-layer isotropic materials $\varepsilon_{A}, \varepsilon_{B}$ are given by:

$$
\begin{aligned}
& \varepsilon_{\mathrm{r}}=\frac{(1+\eta) \varepsilon_{\mathrm{A}} \varepsilon_{\mathrm{B}}}{\varepsilon_{\mathrm{B}}+\eta \varepsilon_{\mathrm{A}}} \\
& \varepsilon_{\theta}=\frac{\varepsilon_{\mathrm{A}}+\eta \varepsilon_{\mathrm{B}}}{1+\eta}
\end{aligned}
$$

in which, $\eta=d_{B} / d_{A}, \quad d_{A}$ and $d_{B}$ are the thicknesses of the layers $A$ and $B$, respectively. For the permittivity these equations correspond to series and parallel addition of capacitances, respectively.

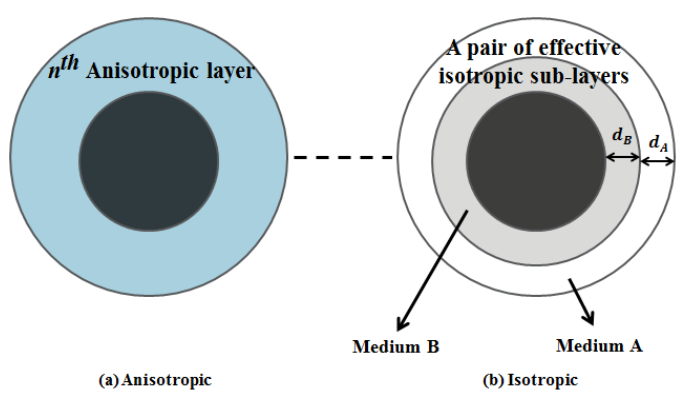

Fig. 4. Equivalence of an anisotropic spherical shell and two isotropic sub-shells

By solving the above equations for $\varepsilon_{A}$ and $\varepsilon_{B}$, one can obtain the equivalent medium parameters for the isotropic sub-layers when the thicknesses are not identical:

$$
\begin{aligned}
& \varepsilon_{B}=\frac{\left[(\eta-1) \varepsilon_{\mathrm{r}}+(1+\eta) \varepsilon_{\theta}\right] \pm \sqrt{\left[(\eta-1) \varepsilon_{\mathrm{r}}+(1+\eta) \varepsilon_{\theta}\right]^{2}-4 \eta^{2} \varepsilon_{\theta} \varepsilon_{\mathrm{r}}}}{2 \eta} \\
& \varepsilon_{\mathrm{A}}=\frac{\left[(1+\eta) \varepsilon_{\theta}-(\eta-1) \varepsilon_{\mathrm{r}}\right] \mp \sqrt{\left[(\eta-1) \varepsilon_{\mathrm{r}}+(1+\eta) \varepsilon_{\theta}\right]^{2}-4 \eta^{2} \varepsilon_{\theta} \varepsilon_{\mathrm{r}}}}{2}
\end{aligned}
$$

For identical thicknesses $(\eta=1)$, 


$$
\begin{aligned}
& \varepsilon_{\mathrm{B}}=\varepsilon_{\theta} \pm \sqrt{\varepsilon_{\theta}{ }^{2}-\varepsilon_{\theta} \varepsilon_{\mathrm{r}}} \\
& \varepsilon_{\mathrm{A}}=\varepsilon_{\theta} \mp \sqrt{\varepsilon_{\theta}^{2}-\varepsilon_{\theta} \varepsilon_{\mathrm{r}}}
\end{aligned}
$$

It is possible to use different combinations for $\varepsilon$ and $\mu$ in the first and second layers, Table 1.

Table 1.Different combinations of $\varepsilon, \mu$

\begin{tabular}{clc}
\hline Case & FirstLayer & Second Layer \\
& & \\
\hline \multirow{2}{*}{ I } & $\varepsilon_{A}=\varepsilon_{\theta}+\sqrt{\varepsilon_{\theta}^{2}-\varepsilon_{\theta} \varepsilon_{r}}$ & $\varepsilon_{B}=\varepsilon_{\theta}-\sqrt{\varepsilon_{\theta}^{2}-\varepsilon_{\theta} \varepsilon_{r}}$ \\
& $\mu_{A}=\mu_{\theta}+\sqrt{\mu_{\theta}^{2}-\mu_{\theta} \mu_{r}}$ & $\mu_{B}=\mu_{\theta}-\sqrt{\mu_{\theta}^{2}-\mu_{\theta} \mu_{r}}$ \\
& & \\
II & $\varepsilon_{A}=\varepsilon_{\theta}+\sqrt{\varepsilon_{\theta}^{2}-\varepsilon_{\theta} \varepsilon_{r}}$ & $\varepsilon_{B}=\varepsilon_{\theta}-\sqrt{\varepsilon_{\theta}^{2}-\varepsilon_{\theta} \varepsilon_{r}}$ \\
& $\mu_{A}=\mu_{\theta}-\sqrt{\mu_{\theta}^{2}-\mu_{\theta} \mu_{r}}$ & $\mu_{B}=\mu_{\theta}+\sqrt{\mu_{\theta}^{2}-\mu_{\theta} \mu_{r}}$ \\
& & \\
III & $\varepsilon_{A}=\varepsilon_{\theta}-\sqrt{\varepsilon_{\theta}^{2}-\varepsilon_{\theta} \varepsilon_{r}}$ & $\varepsilon_{B}=\varepsilon_{\theta}+\sqrt{\varepsilon_{\theta}^{2}-\varepsilon_{\theta} \varepsilon_{r}}$ \\
& $\mu_{A}=\mu_{\theta}+\sqrt{\mu_{\theta}^{2}-\mu_{\theta} \mu_{r}}$ & $\mu_{B}=\mu_{\theta}-\sqrt{\mu_{\theta}^{2}-\mu_{\theta} \mu_{r}}$ \\
& & \\
\hline
\end{tabular}

In case $I$, the impedances in the layers $\sqrt{\mu / \varepsilon}$ are equal, whereas in cases II and III the refractive index $\sqrt{\varepsilon_{\mathrm{r}} \mu_{\mathrm{r}}}$ is equal in the layers. Cases II and III differ only in whether the inner layer has the larger or the smaller value of $\varepsilon$.

\section{Scattering from a Dielectric Sphere with Multilayered Coating}

The configuration for electromagnetic scattering by the spherical body coated by $2 \mathrm{M}$ layers is shown in Fig. 5. The external radius, permittivity, and permeability of the core and the layers are denoted by $\mathrm{a}_{\mathrm{i}}, \varepsilon_{\mathrm{i}}$ and $\mu_{\mathrm{i}}(\mathrm{i}=1,2, . ., 2 \mathrm{M}+1)$, respectively. The outer radius of each sub-layer in the physical space is given by Eqns. 6, 10. The values of the anisotropic parameters are used at the center of each anisotropic layer, i.e. for the even values of $i$ [16].

Fig. 5 shows an $E_{x}$ polarized plane wave with amplitude $E_{0}, E^{i}=E_{o} e^{-j K_{0} z} \bar{x}$, incident upon the coated sphere along the $\hat{z}$ direction. $\mathrm{k}_{0}=\omega \sqrt{\mu_{0} \varepsilon_{0}}$ is the wave number in free space. The time dependence $\mathrm{e}^{\mathrm{j} \omega \mathrm{t}}$ is suppressed.

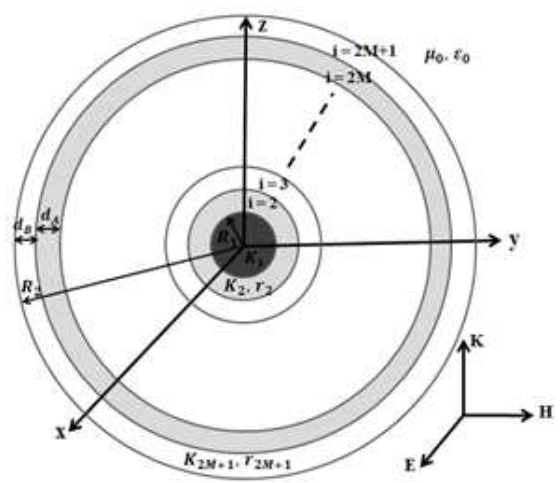

Fig.5. Plane wave scattering by a multilayer dielectric sphere

The fields in the different regions are expanded in terms of spherical harmonics of $T E_{r}$ and $T M_{r}$ modes w.r.t. the radial direction. The field ( $E$ or $H$ ) with only transversecomponents $(\theta, \varnothing)$ is expressed by the harmonics $m$, whereas the other field having the three components is expressed by the harmonics $n$ [25], [26].

$$
\mathrm{m}_{\mathrm{e}}^{\mathrm{o}}= \pm \frac{1}{\sin \theta} \mathrm{J}_{\mathrm{n}}(\mathrm{kr}) \mathrm{P}_{\mathrm{n}}^{1}(\cos \theta){ }_{\sin }^{\cos } \emptyset \hat{\theta}-\mathrm{J}_{\mathrm{n}}(\mathrm{kr}) \frac{\partial \mathrm{P}_{\mathrm{n}}^{1}}{\partial \theta} \sin \phi \widehat{c o s}
$$

$\mathrm{n}_{\mathrm{e}}^{\mathrm{o}}=$

$\frac{\mathrm{n}(\mathrm{n}+1)}{\mathrm{kr}} \mathrm{J}_{\mathrm{n}}(\mathrm{kr}) \mathrm{P}_{\mathrm{n}}^{1}(\cos \theta) \cos _{\cos }^{\sin } \emptyset \hat{\mathrm{r}}+$

$\frac{1}{\mathrm{kr}}\left[\mathrm{krJ}_{\mathrm{n}}(\mathrm{kr})\right]^{\prime} \frac{\partial \mathrm{P}_{\mathrm{n}}^{1}}{\partial \theta} \sin \cos \phi \hat{\theta}_{-}^{+} \frac{1}{\mathrm{krsin} \theta}\left[\mathrm{krJ}_{\mathrm{n}}(\mathrm{kr})\right]^{\prime} \mathrm{P}_{\mathrm{n}}^{1}(\cos \theta)_{\sin }^{\cos } \emptyset \widehat{\emptyset}$

where $\mathrm{J}_{\mathrm{n}}(\mathrm{kr})$ is a spherical Bessel function of order $\mathrm{n}$ of any kind and $\mathrm{P}_{\mathrm{n}}^{1}(\cos \theta)$ is a Legendre polynomial of the first kind, first order and $n^{\text {th }}$ degree. The $\emptyset$-dependnce is taken as $\sin \emptyset$ or $\cos \emptyset$ (odd, o, or even, e) to conform with the incident plane wave.

The incident plane wave can be expressed in terms of spherical harmonics with Bessel functions of the first kind as:

$$
\begin{gathered}
E^{i}=E_{o} e^{-j K_{0} z} \bar{x}=\sum_{n=1} G_{n}\left(m_{o}^{i}+j n_{e}^{i}\right) \\
H^{i}=-\sum_{n=1} \frac{G_{n}}{Z_{0}}\left(m_{e}^{i}-j n_{o}^{i}\right)
\end{gathered}
$$

where

$$
G_{n}=(-j)^{n} \frac{2 n+1}{n(n+1)} E_{o}
$$

and $Z_{0}=\sqrt{\frac{\mu_{0}}{\varepsilon_{0}}}, j=\sqrt{-1}$

The scattered fields can be expanded in terms of spherical harmonics with spherical Hankel functions $\mathrm{h}_{\mathrm{n}}^{2}\left(\mathrm{k}_{0} \mathrm{r}\right)$ representing scattered outgoing waves with unknown coefficients $\mathrm{e}^{\mathrm{s}}, \mathrm{d}^{\mathrm{s}}$ of the TE, TM parts, respectively.

$$
\begin{aligned}
E^{s} & =\sum_{n=1} G_{n}\left(e^{s} m_{o}^{s H}+j d^{s} n_{e}^{s H}\right) \\
H^{s} & =-\sum_{n=1} \frac{G_{n}}{z_{0}}\left(d^{s} m_{e}^{s H}-j e^{s} n_{o}^{s H}\right)
\end{aligned}
$$

We can write the fields in the $\mathrm{i}^{\text {th }}$ Layer as:

$$
\begin{gathered}
E^{c i}=\sum_{n=1} G_{n}\left(d^{c H^{1} i} m_{o}^{c H^{1}}+j e^{c H^{1} i} n_{e}^{c H^{1}}+d^{c H^{2} i} m_{o}^{c H^{2}}+\right. \\
\mathrm{H}^{\mathrm{ci}}=-\sum_{\mathrm{n}=1} \frac{\mathrm{G}_{\mathrm{n}}}{\mathrm{z}_{0}}\left(\mathrm{e}^{\mathrm{cH} \mathrm{H}^{1} \mathrm{i}} \mathrm{m}_{\mathrm{e}}^{\mathrm{cH}}-\mathrm{j} \mathrm{d}^{\mathrm{cH}{ }^{1} \mathrm{i}} \mathrm{n}_{0}^{\mathrm{cH}}+\mathrm{e}^{\mathrm{CH}^{2} \mathrm{i}} \mathrm{m}_{\mathrm{e}}^{\mathrm{cH}}-\right. \\
\left.\mathrm{j} \mathrm{d}^{\mathrm{cH}^{2} \mathrm{i}} \mathrm{n}_{\mathrm{o}}^{\mathrm{cH}}\right)
\end{gathered}
$$

where the modes in the cloak region are represented by Hankel functions of the first and second kinds with arguments $\left(\mathrm{k}_{\mathrm{i}} \mathrm{r}_{\mathrm{i}}\right)$, where $\mathrm{k}_{\mathrm{i}}=\omega \sqrt{\mu_{\mathrm{i}} \epsilon_{\mathrm{i}}}$, and unknown coefficients $\mathrm{d}$,e. The boundary conditions at the interface between layers $i, i+1$ lead to two equations relating the TM coefficients $\mathrm{d}^{\mathrm{cH}^{1}}, \mathrm{~d}^{\mathrm{cH}^{2}}$ of the two layers, and two equations relating the $\mathrm{TE}$ coefficients $\mathrm{e}^{\mathrm{cH}^{1}}, \mathrm{e}^{\mathrm{cH}^{2}}$ of the two layers. The finiteness of the field in the dielectric core leads to thefollowing ratios in the dielectric core [27]: 


$$
\frac{d^{c H^{2}(1)}}{d^{c H^{1}(1)}}=1 \quad, \frac{e^{c H^{2}(1)}}{e^{c H^{1}(1)}}=1
$$

When cloaking a conducting sphere, the dielectric constant in the core is taken to be very large.

The ratios $d^{c H^{2}(i)} / d^{c H^{1}(i)}$ and $e^{c H^{2}(i)} / e^{c H^{1}(i)}$ in the successive larger layers can be obtained iteratively from the following equations [27]:

$$
\begin{aligned}
& \frac{d^{c H^{2}(i+1)}}{d^{c H^{1}(i+1)}}=-\frac{H_{n}^{1}\left(K_{i+1} r_{i}\right)-R_{H}^{i} H_{n}^{1 \prime}\left(K_{i+1} r_{i}\right)}{H_{n}^{2}\left(K_{i+1} r_{i}\right)-R_{H}^{i} H_{n}^{2 \prime}\left(K_{i+1} r_{i}\right)}, \mathrm{i}=1,2, \ldots 2 \mathrm{M} \\
& \frac{e^{c H^{2}(i+1)}}{e^{c H^{1}(i+1)}}=-\frac{H_{n}^{1}\left(K_{i+1} r_{i}\right)-R_{E}^{i} H_{n}^{1 \prime}\left(K_{i+1} r_{i}\right)}{H_{n}^{2}\left(K_{i+1} r_{i}\right)-R_{E}^{i} H_{n}^{2 \prime}\left(K_{i+1} r_{i}\right)}, \mathrm{i}=1,2, \ldots 2 \mathrm{M}
\end{aligned}
$$

where $\mathrm{H}_{\mathrm{n}}^{1}(\mathrm{KR})=\mathrm{KRh}_{\mathrm{n}}^{1}(\mathrm{KR})$ and $\mathrm{H}_{\mathrm{n}}^{2}(\mathrm{KR})=\mathrm{KRh}_{\mathrm{n}}^{2}(\mathrm{KR})$ are the Riccati-Hankel functions.

$$
\begin{aligned}
& R_{H}^{i}=\sqrt{\frac{\mu_{i+1} \epsilon_{i}}{\epsilon_{i+1} \mu_{i}}} \frac{H_{n}^{1}\left(K_{i} r_{i}\right)+\frac{d^{c H^{2}(i)}}{d^{c H^{1}(i)}} H_{n}^{2}\left(K_{i} r_{i}\right)}{H_{n}^{1 \prime}\left(K_{i} r_{i}\right)+\frac{d^{c H^{2}(i)}}{d^{c H^{1}(i)}} H_{n}^{2 \prime}\left(K_{i} r_{i}\right)}, \mathrm{i}=1,2, \ldots 2 \mathrm{M}(31) \\
& R_{E}^{i}=\sqrt{\frac{\mu_{i} \epsilon_{i+1}}{\epsilon_{i} \mu_{i+1}}} \frac{H_{n}^{1}\left(K_{i} r_{i}\right)+\frac{e^{c H^{2}(i)}}{e^{c H^{1}(i)}} H_{n}^{2}\left(K_{i} r_{i}\right)}{H_{n}^{1 \prime}\left(K_{i} r_{i}\right)+\frac{e^{c H^{2}(i)}}{e^{c H^{1}(i)}} H_{n}^{2 \prime}\left(K_{i} r_{i}\right)}, \mathrm{i}=1,2, \ldots 2 \mathrm{M}(32)
\end{aligned}
$$

Finally, the boundary conditions between the outer layer and air lead to the scattering coefficients $b_{n}=d^{s}$ (TM part) and $\mathrm{a}_{\mathrm{n}}=\mathrm{e}^{\mathrm{s}}$ (TE part):

$$
\begin{gathered}
b_{n}=-\frac{j_{n}\left(K_{0} R_{2}\right)-R_{H}^{2 M+1} j_{n}^{\prime}\left(K_{0} R_{2}\right)}{H_{n}^{2}\left(K_{0} R_{2}\right)-R_{H}^{2 M+1} H_{n}^{2 \prime}\left(K_{0} R_{2}\right)} \\
a_{n}=-\frac{j_{n}\left(K_{0} R_{2}\right)-R_{E}^{2 M+1} j_{n}^{\prime}\left(K_{0} R_{2}\right)}{H_{n}^{2}\left(K_{0} R_{2}\right)-R_{E}^{2 M+1} H_{n}^{2^{\prime}}\left(K_{0} R_{2}\right)}
\end{gathered}
$$

The scattering cross section $\sigma_{\mathrm{s}}$ and the normalized radar cross sections $Q_{(\theta, \varphi)}$ are given by [28], [29]:

$$
\begin{gathered}
\sigma_{\mathrm{s}}=4 \pi \mathrm{r}^{2} \frac{\left|\mathrm{E}^{\mathrm{S}}\right|^{2}}{\left|\mathrm{E}^{\mathrm{i}}\right|^{2}} \\
\mathrm{Q}_{(\theta, \varphi)}=\frac{\sigma_{\mathrm{s}}}{\pi R_{1}{ }^{2}}=\frac{4}{\left(K_{0} R_{1}\right)^{2}}\left(\left|\mathrm{~S}_{1}(\theta)\right|^{2} \sin ^{2} \varphi+\left|\mathrm{S}_{2}(\theta)\right|^{2} \cos ^{2} \varphi\right)
\end{gathered}
$$

where $S_{1}(\theta)$ and $S_{2}(\theta)$ are defined by :

$$
\begin{aligned}
& S_{1}(\theta)=\sum_{n} \frac{(2 n+1)}{n(n+1)}\left[a_{n} \pi_{n}(\theta)+b_{n} \tau_{n}(\theta)\right] \\
& S_{2}(\theta)=\sum_{n} \frac{(2 n+1)}{n(n+1)}\left[b_{n} \pi_{n}(\theta)+a_{n} \tau_{n}(\theta)\right]
\end{aligned}
$$

In the above two equations $\pi_{n}(\theta)$ and $\tau_{n}(\theta)$ describe the angular scattering patterns of the spherical harmonics used to describe $S_{1}$ and $S_{2}$ and follow from the recurrence relations [29], [30]:

$$
\begin{gathered}
\pi_{n}=\frac{P_{n}^{1}(\cos \theta)}{\sin \theta}=\frac{2 n-1}{n-1} \cos \theta \cdot \pi_{n-1}-\frac{n}{n-1} \pi_{n-2} \\
\tau_{n}=\frac{d P_{n}^{1}(\cos \theta)}{d \theta}=n \cos \theta \cdot \pi_{n}-(n+1) \pi_{n-1}
\end{gathered}
$$

starting with the initial values: $\pi_{0}=0 ; \pi_{1}=1$.

The total scattering normalized cross section $Q_{\text {sca }}$ follows from the integration of the scattered power over all directions $\theta, \varphi$, given by [31]:

$$
\mathrm{Q}_{\mathrm{sca}}=\frac{2}{\left(K_{0} R_{1}\right)^{2}} \sum_{\mathrm{n}=1}^{\infty}(2 \mathrm{n}+1)\left(\left|\mathrm{a}_{\mathrm{n}}\right|^{2}+\left|\mathrm{b}_{\mathrm{n}}\right|^{2}\right)
$$

The backscattering normalized radar cross section $Q_{b}$, applicable to monostatic radar, is given by [31]:

$$
\mathrm{Q}_{\mathrm{b}}=\frac{1}{\left(K_{0} R_{1}\right)^{2}}\left|\sum_{\mathrm{n}=1}^{\infty}(-1)^{\mathrm{n}}(2 \mathrm{n}+1)\left(\mathrm{a}_{\mathrm{n}}-\mathrm{b}_{\mathrm{n}}\right)\right|^{2}
$$

The mode series is truncated at the mode number $\mathrm{n}_{\max }=\mathrm{K}_{0} \mathrm{R}_{2}+4 *\left(\mathrm{~K}_{0} \mathrm{R}_{2}\right)^{1 / 3}+2,[29],[30]$.

\section{Results}

\subsection{Scattering from cloaked Perfect Electrically Conducting (PEC) Sphere}

\subsubsection{Effect of Arrangement of Materials in the Layers}

To show the effect of different cases of arrangement of materials in the layers, table 1 , on the bistatic radar cross section $\sigma_{\mathrm{s}}$ (RCS), consider the inner core with radius $\mathrm{R}_{1}=\lambda$, the outermost radius $\mathrm{R}_{2}=2 \lambda$, where $\lambda$ is the wavelength, and the operating frequency $f$ is $2 \mathrm{GHz}$, the cloak is discretized into $2 \mathrm{M}=80$ Layers (these values are used for the bistatic scattering in the paper unless otherwise specified). Fig. 6 shows the bistaic radar cross section area (RCS) of the concave-up nonlinear cloaks versus $\theta$ of the multilayered isotropic structure for the ideal case $(\mathrm{c}=0)$ for the three different arrangement cases and the nonlinearity degree $\mathrm{x}=0.1$. The results for cases I and II are checked against ref. [16] with identical results. The main difference between cases II and III are near the forward direction $(\theta=0)$.

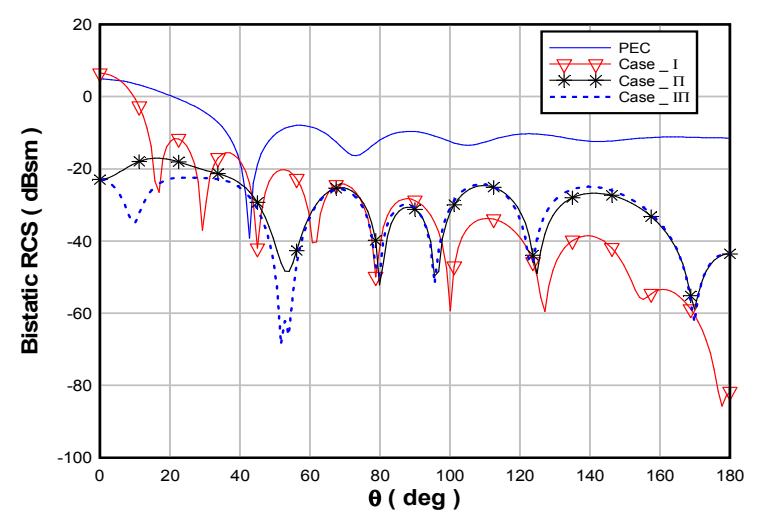

Fig. 6. Bistatic RCS for the concave-up nonlinear ideal cloaks ( $c=0), x=$ O.1for different cases

It can be seen from Fig. 6 that the backscattering $\left(\theta=180^{\circ}\right)$ in cases II and III has not been reduced to the same level as that in case I. Case I leads to proper impedance matching of the layers but leads to large jumps of therefractive index (equal to the square root of the product of $\varepsilon, \mu)$ in the successive layers. This leads to low back scattering, but high forward scattering[20]. On the other 
hand, the second and third approaches lead to gradual variation of the refractive index in the successive sub-layers, but the impedance of the successive sub-layers suffers from jumps [23]. This increases the back scattering but improves the forward and total scattering. In the near forward angles, the scattering from case III is smaller than case II.

Fig. 7 shows the bistaic RCS of the concave-down nonlinear ideal cloaks $(\mathrm{c}=0)$ for cases II and III and the nonlinearity degree $\mathrm{x}=0.1$. For $\mathrm{x}=0.1$ for concave-down cloak, the results for cases II, III are nearly the same as the corresponds results for concave-up cloak (Fig. 6).

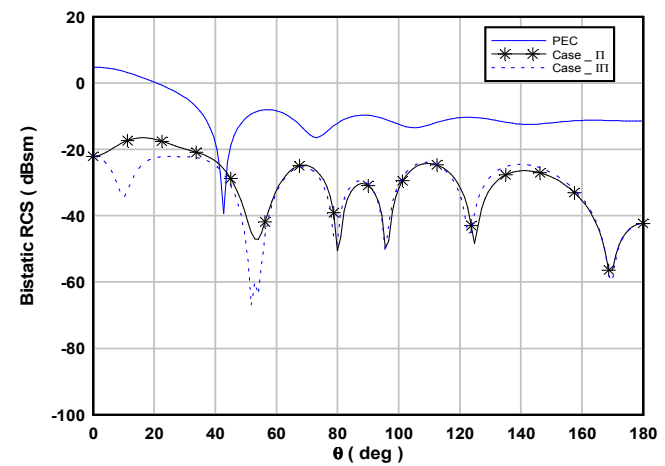

Fig. 7. Bistatic RCS for the concave-down nonlinear ideal cloaks for different cases

\subsubsection{Effect of the Reduced Radius $c$}

Figs. 8, 9 and 10 show the bistaic RCS of the concave-up nonlinear cloaks (case II) for different values of approximate cloaking radius $\mathrm{c}$ and the nonlinearity degrees $\mathrm{x}=0.1,0.6,20$, respectively. The forward scattering is nearly identical for $c=\lambda / 10$ to $c=\lambda / 40$. The back scattering improves for $c=\lambda / 20, \lambda / 40$ for $\mathrm{x}=0.1,0.6$ than for $x=20$. The value of the back scattering depends actually on whether the scattering pattern ends in the back scattering direction with a peak or trough. As the transformed radius $\mathrm{c}$ increases to $\lambda / 5$, the scattering increases as shown in Fig. 9.For the ideal profile $(\mathrm{c}=0)$, $\varepsilon_{\mathrm{r}} / \varepsilon_{0}=0$ at $\mathrm{r}=\mathrm{R}_{1}$, Eqn. 8. As c increases, $\varepsilon_{\mathrm{r}} / \varepsilon_{0}$ at $\mathrm{r}=\mathrm{R}_{1}$ increases. For $\mathrm{c}=\mathrm{R}_{1} / 40, \mathrm{x}=1, \varepsilon_{\mathrm{r}} / \varepsilon_{0} \cong 1 / 3200$. For $\mathrm{c}=\mathrm{R} 1 / 10, \mathrm{x}=1, \varepsilon_{\mathrm{r}} / \varepsilon_{0} \cong 1 / 200$.

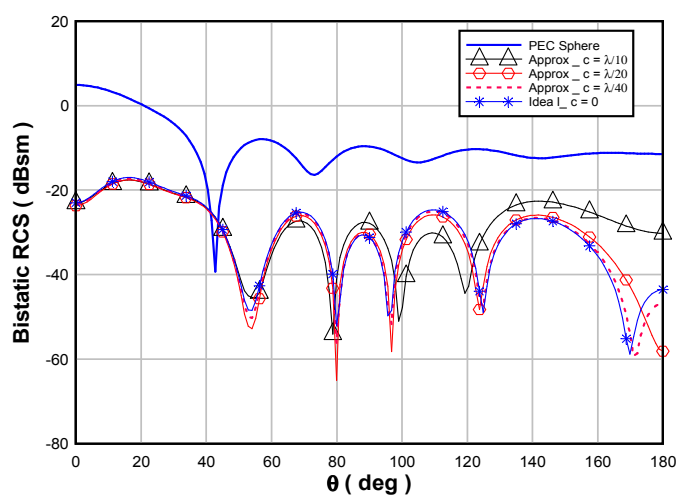

Fig. 8. Bistatic RCS for the concave-up nonlinear cloaks for different values of the cloaking radii $c$ and $x=0.1$ (case II)

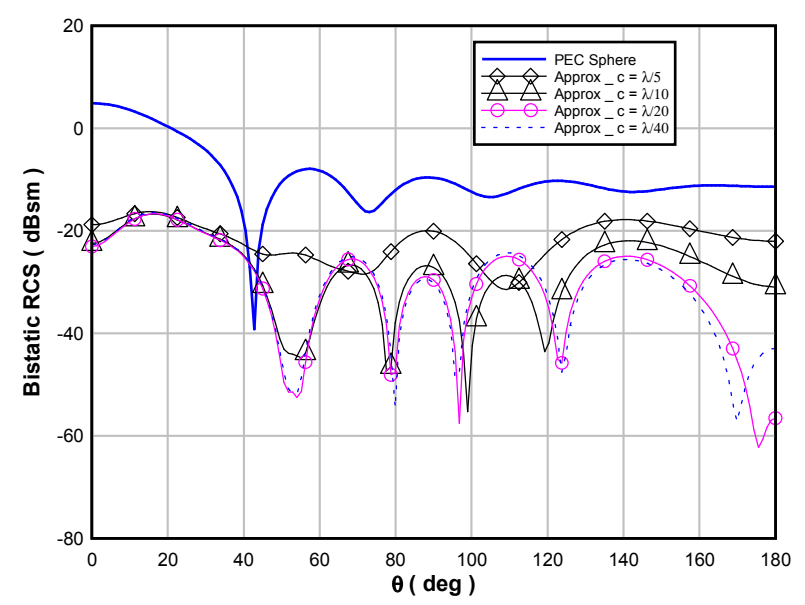

Fig. 9. Bistatic RCS for the concave-up nonlinear cloaks for different values of the cloaking radii $c$ and $x=0.6$ (case II)

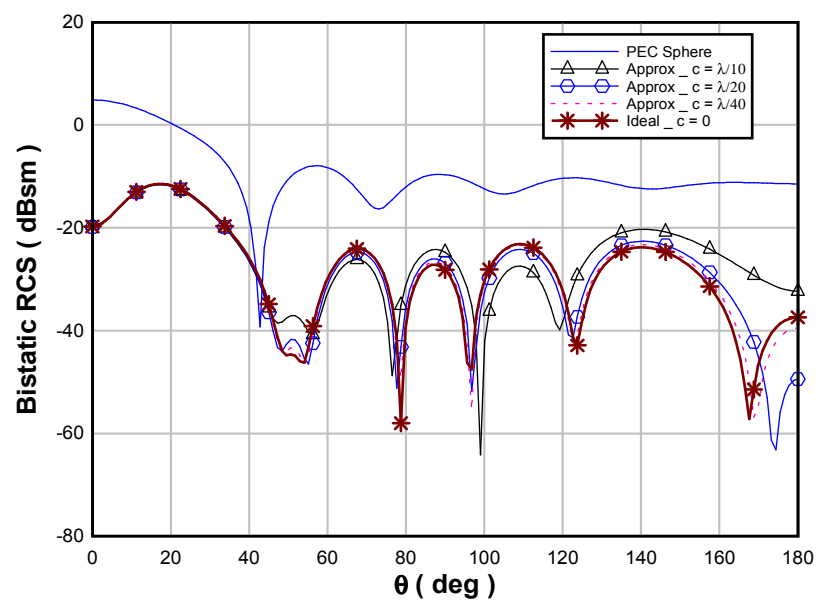

Fig. 10. Bistatic RCS for the concave-up nonlinear cloaks for different values of the cloaking radii $c$ and $x=20$ (case II)

Figs. 11,12 and 13 show the bistaic RCS of the concave-up nonlinear cloaks ( case III ) for different values of approximate cloaking radius $\mathrm{c}$ and the nonlinearity degree $x=0.1,0.6,20$, respectively.

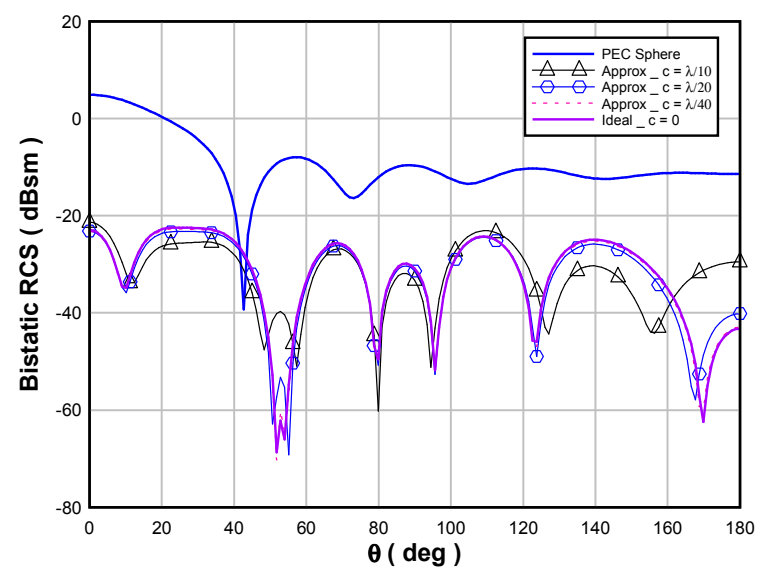

Fig. 11. Bistatic RCS for the concave-up nonlinear cloaks for different values of the cloaking radii $c$ and $x=0.1$ (case III) 


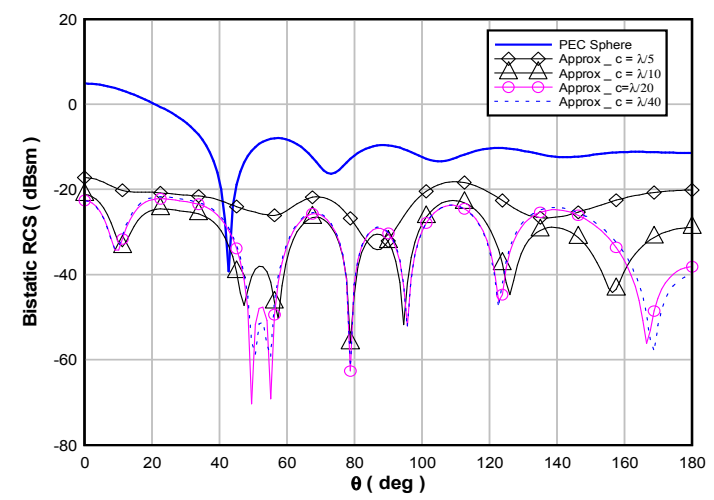

Fig. 12. Bistatic RCS for the concave-up nonlinear cloaks for different values of the cloaking radii $c$ and $x=0.6$ (case III)

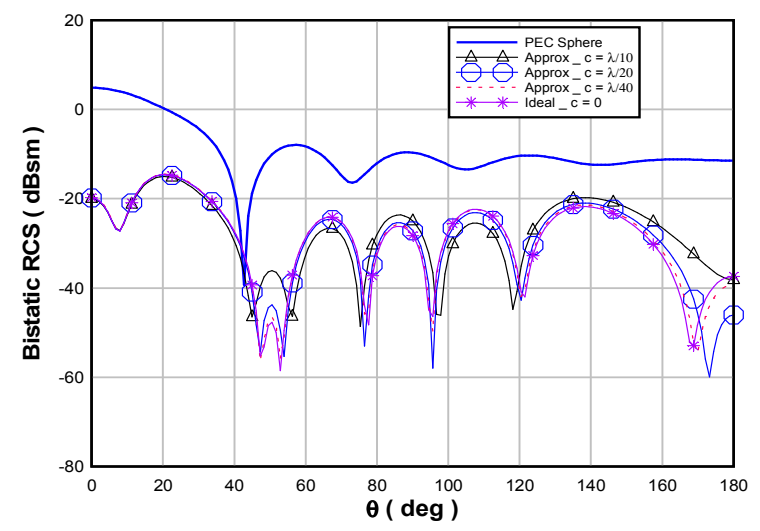

Fig. 13. Bistatic RCS for the concave-up nonlinear cloaks for different values of the cloaking radii $c$ and $x=20$ (case III)

\subsubsection{Effect of the Nonlinearity Degree $x$}

Here, we will study the effects of the nonlinearity degree $\mathrm{x}$ on the scattering from nonlinear spherical cloaks. We fix $\mathrm{c}=\mathrm{R}_{1} / 40$, and consider $\mathrm{x}=0.1,0.6,1,5,10$ and $\mathrm{x}=20$ for concave-down and concave-up nonlinear cloaks, as discussed in the following figures.

Figs. 14 and 15 show the bistaic RCS of the concave-down nonlinear cloaks for both cases II and III for six different values of the nonlinearity degree $\mathrm{x}$.

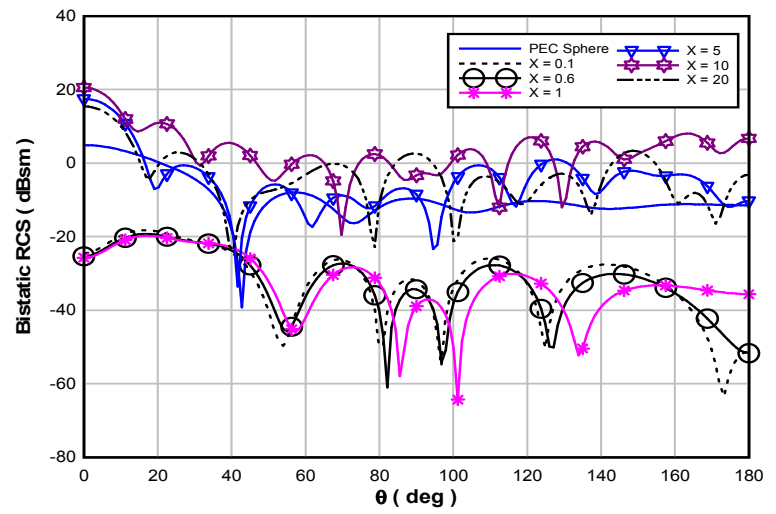

Fig. 14. Bistatic RCS for the concave-down nonlinear approximate cloaking for different values of $x$ (case II)

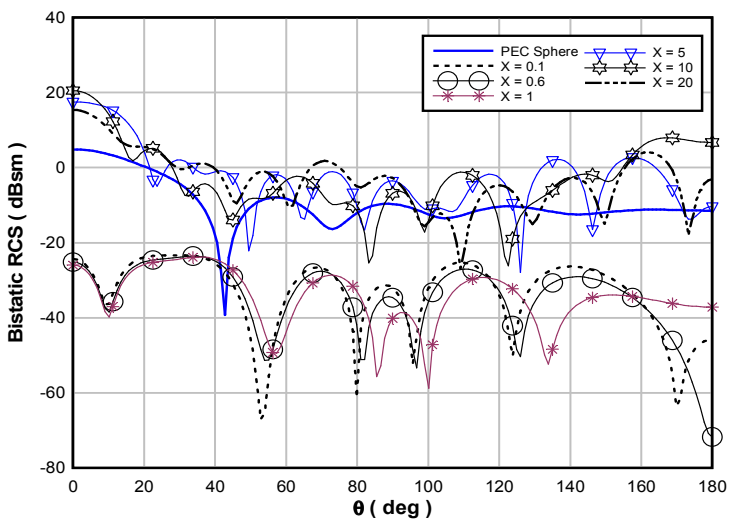

Fig. 15. Bistatic RCS for the concave-down nonlinear approximate cloaking for different values of $x$ (case III)

From Figs. 14 and 15, it can be seen that for concave-down nonlinear transformations, the cloaking property is better retained when $\mathrm{x}<1$. As $\mathrm{x}$ increases $(x>1)$, their RCSs are increasing dramatically and can be larger than that of an uncloaked PEC core (e.g., $x=5,10$ and 20), which is not desired in the cloaking application [15], [16].

Figs. 16 and 17 show the bistaic RCS of the concave-up nonlinear cloaks for both cases II and III for different values of the nonlinearity degree $\mathrm{x}$. The value of the exponent $\mathrm{x}$ has much less effect on the scattering than in the concave down case. The back scattering in concave up case is higher than in the concave down case for $\mathrm{x}<1$, Figs. 14-17.

The increase of $\mathrm{x}$ in concave-up transformations will push the "hot" areas (region with sharp variations of the electrical properties, Figs. 2, 3) further and further away from the spherical core, so the far-field pattern become stable with increasing $x$. However, the increase of $x$ in the concave-down transformations has little effect in shifting away the "hot" positions from the core, which results in larger interaction with the spherical core. Hence, the RCS reduction in the far field is degraded with the increase of $x$, where the variations of the electrical properties become sharper in the concave-down class as shown in the figures [15], [16].

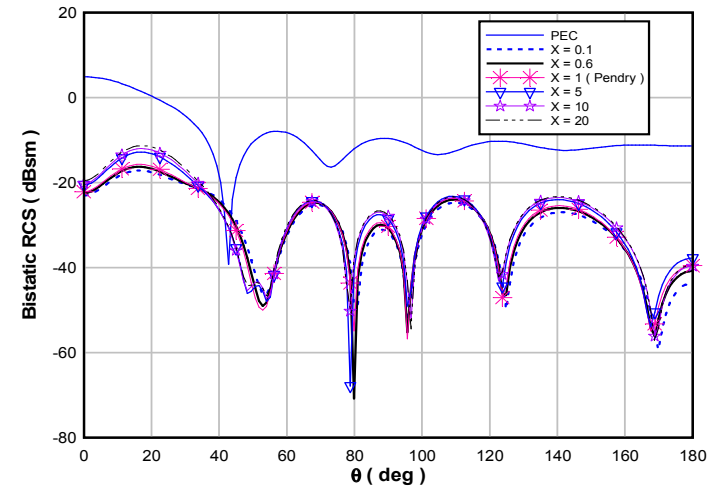

Fig. 16. Bistatic RCS for the concave-up nonlinear cloaks for different values of $x$ (case II) 


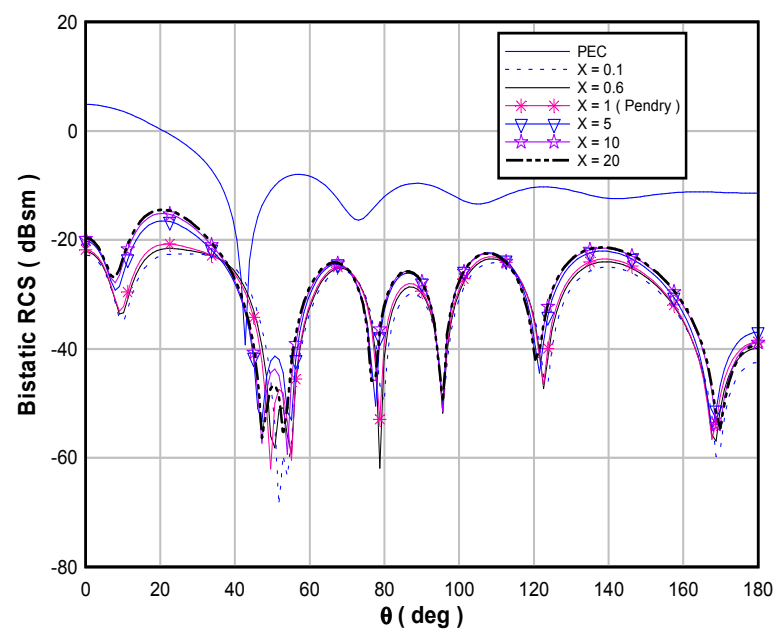

Fig. 17. Bistatic RCS for the concave-up nonlinear cloaks for different values of $x$ (case III)

\subsubsection{Effect of Frequency and Reduced Radius c}

Figs. 18, 19 show the back scattering RCS of the concave-up and concave-down nonlinear cloaks against the normalized frequency $\mathrm{R}_{1} / \lambda$ for different values of $c$ (case II) at $2 \mathrm{M}=80, \mathrm{R}_{1}=0.15 \mathrm{~m}, \mathrm{R}_{2}=2 \mathrm{R}_{1}$ (these values are used in the paper for the back scattering versus frequency, unless otherwise specified) and for $\mathrm{x}=0.6$.

The average of the back scattering with frequency increases with increasing frequency. This is because with a fixed cloak thickness and number of layers the layer thickness becomes a larger fraction of the wavelength with increasing frequency. For concave-up cloak with $\mathrm{c}=$ $\mathrm{R}_{1} / 40$, Fig. 18 , some scattering peaks appear due to interactions between the layers.

The concave-down cloak, Fig. 19, shows lower scattering than the concave-up cloak, Fig. 18.

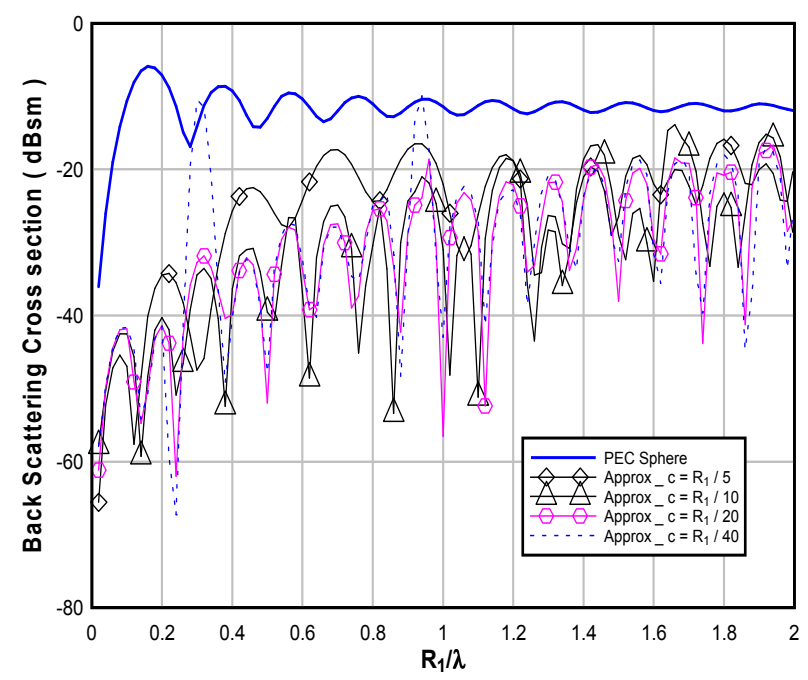

Fig. 18. The back scattering RCS for the concave-up nonlinear cloaks for different values of $c$ (case II) at $x=0.6$

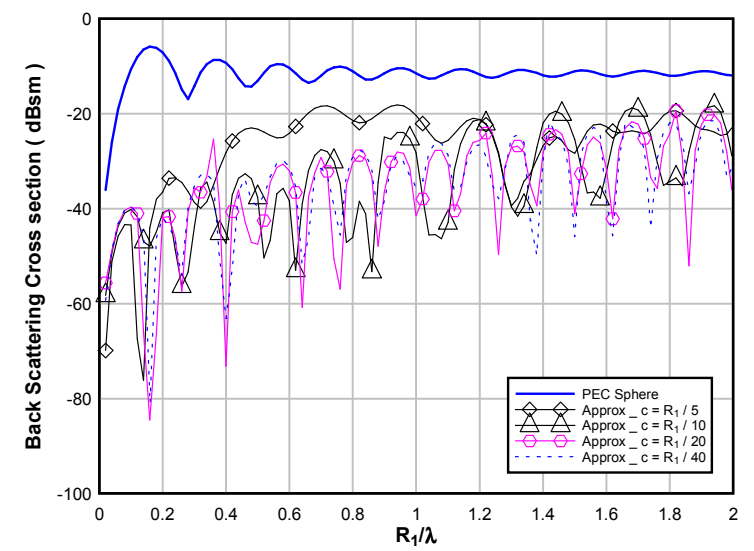

Fig. 19. The back scattering RCS for the concave-down nonlinear cloaks for different values of $c$ (case II) at $x=0.6$

\subsubsection{Effect of Frequency and Nonlinearity Degree $x$}

The scattering properties of an approximate cloaked conducting sphere coated with isotropic homogenous layers are investigated concerning the back scattering cross section versus the normalized frequency $R_{1} / \lambda$ for different values of the nonlinearity degree $x$.

Fig. 20 shows the back scattering RCS of the concave-down nonlinear cloaks versus the normalized frequency for different values of $x$ (case II) at $c=R_{1} / 40$. Fig. 21 shows the corresponding results for case III.

At most frequencies, the cloak with $\mathrm{x}=1$ show least scattering. It can be seen, from Figs. 20 and 21 for concave -down profile that when the nonlinearity degree $\mathrm{x}$ becomes larger than unity, the scattering increase compared to the uncoated conducting sphere, which is useless for cloaking.

Fig. 22 shows the back scattering RCS of the concave-up nonlinear cloaks for different values of $x$ (case II) at $c=R_{1} / 40$. The value of the exponent $x$ has much less effect on scattering compared with the concave-down profile. In Fig. 22 large values of back scattering at some frequencies and exponent $\mathrm{x}$ are found due to interaction effects between the layers, corresponding to those found in Fig. 18.

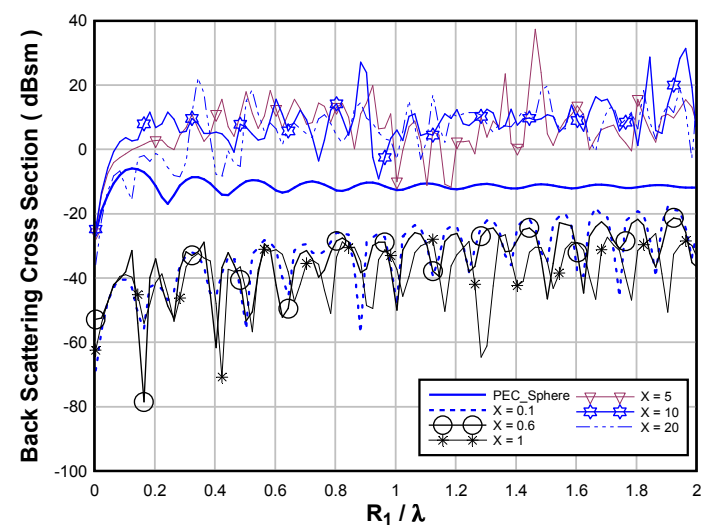

Fig. 20. The back scattering RCS for the concave-down nonlinear cloaks for different values of $x$ (case II) at $c=R_{1} / 40$ 


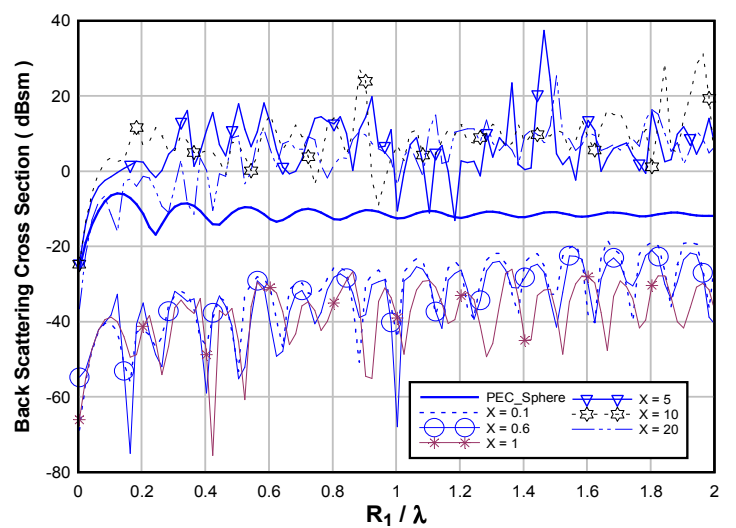

Fig. 21. The back scattering RCS for the concave-down nonlinear cloaks for different values of $x$ (case III) at $c=R_{1} / 40$

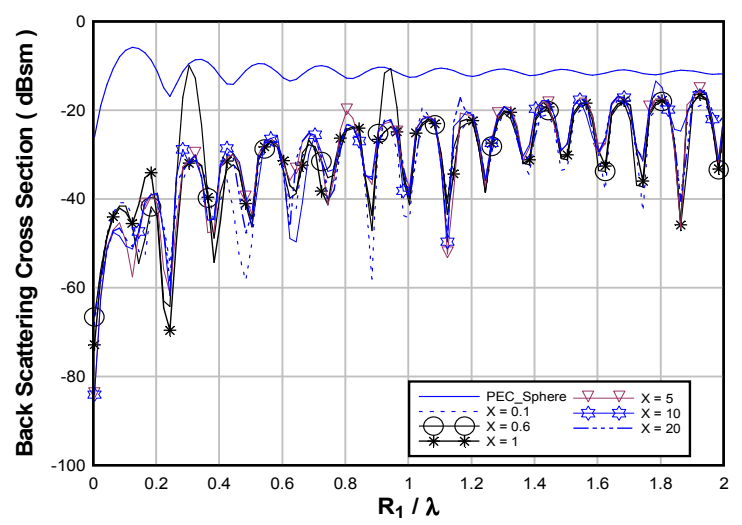

Fig. 22. The back scattering RCS for the concave-up nonlinear cloaks for different values of $x$ (case II) at $c=R_{1} / 40$

\subsection{Scattering from cloaked dielectric Sphere}

\subsubsection{Effect of Dielectric Constant and Layers Configurations}

Fig. 23 shows the bistaic RCS of the concave-up nonlinear cloaks for the ideal case $(\mathrm{c}=0)$ for cases II and III for a dielectric sphere with $\varepsilon_{\mathrm{d}}=2$ and the nonlinearity degree $\mathrm{x}=0.1$. The results are nearly identical to the cloaked conducting sphere, Fig. 6 , due to near perfect cloaking for $\mathrm{c}=0$. The results for a sphere with $\varepsilon_{\mathrm{d}}=10$ are nearly identical.

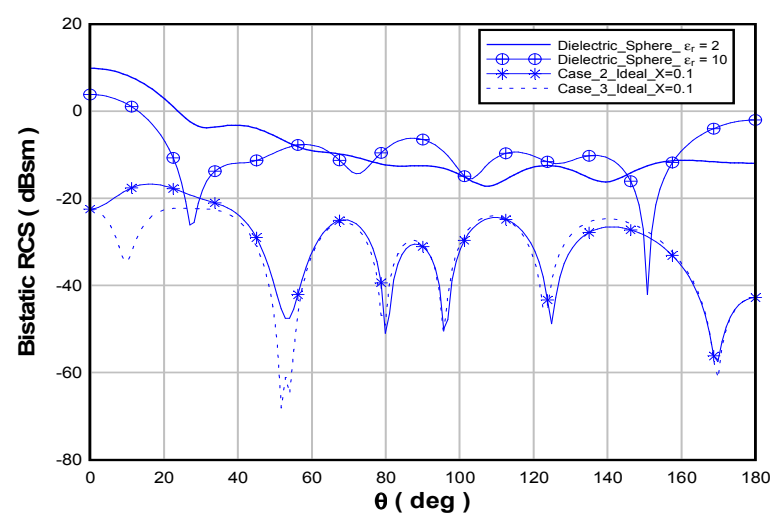

Fig. 23. Bistatic RCS for the concave-up nonlinear ideal cloaks for cloaked dielectric sphere with $\varepsilon_{d}=2$
Fig. 24 shows the bistaic RCS of the concave-down nonlinear cloaks for the ideal case $(\mathrm{c}=0)$ for cases II and III for a dielectric sphere with $\varepsilon_{\mathrm{d}}=2$ and the nonlinearity degree $\mathrm{x}=0.1$. The scattering is nearly identical to the cloaked conducting sphere, Fig. 7, with slight difference for back scattering. This shows that the cloaking of the body is nearly ideal and the scattering properties are mainly a result of the scattering from the cloaking layers. Similar behavior is also found for $\varepsilon_{\mathrm{d}}=10$.

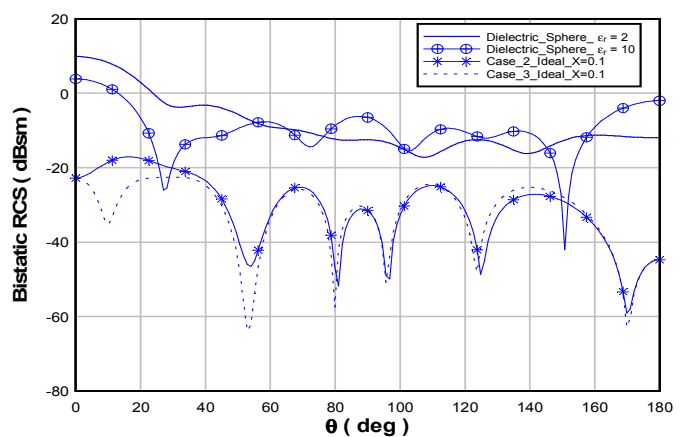

Fig. 24. Bistatic RCS for the concave-down nonlinear ideal cloaks for cloaked dielectric sphere with $\varepsilon_{d}=10$

\subsubsection{Effect of the Reduced Radius $c$}

Figs. 25, 26 show the effect of the reduced radius $\mathrm{c}$ on the bistaic RCS of the concave-up and concave-down nonlinear cloaks, respectively, for case II for cloaked dielectric sphere with $\varepsilon_{\mathrm{d}}=10$ and the nonlinearity degree $\mathrm{x}=0.6\left(\mathrm{R}_{1}=\lambda\right.$, $R_{2}=2 R_{1}$ ). The scattering increases for $c=\lambda / 10, \lambda / 5$.

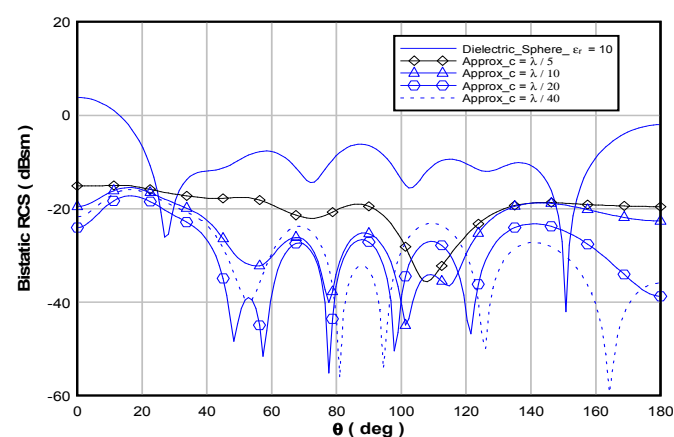

Fig. 25. Bistatic RCS for the concave-up nonlinear approximate cloaking for different values of the cloaking radii $c$ and $x=0.6$ for cloaked dielectric sphere with $\varepsilon_{d}=10$ (case II)

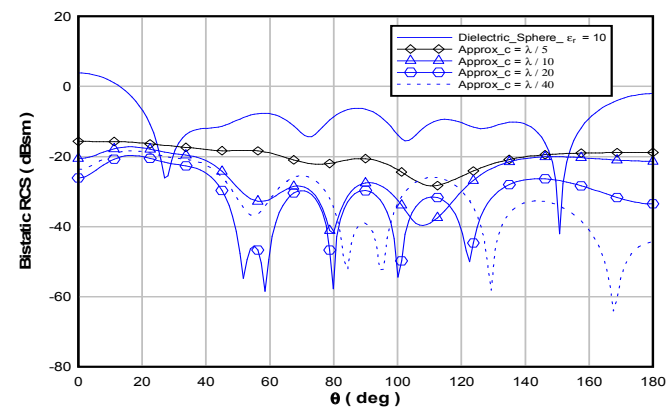

Fig. 26. Bistatic RCS for the concave-down nonlinear approximate cloaking for different values of the cloaking radii $c$ and $x=0.6$ for cloaked dielectric sphere with $\varepsilon_{d}=10$ (case II) 
Figs. 27, 28 show the corresponding results for case III for the bistaic RCS of the concave-up and concave-down nonlinear cloaks, respectively. The back scattering for the concave-down profile, Fig. 28, is lower than that of the concave-up profile, Fig. 27.

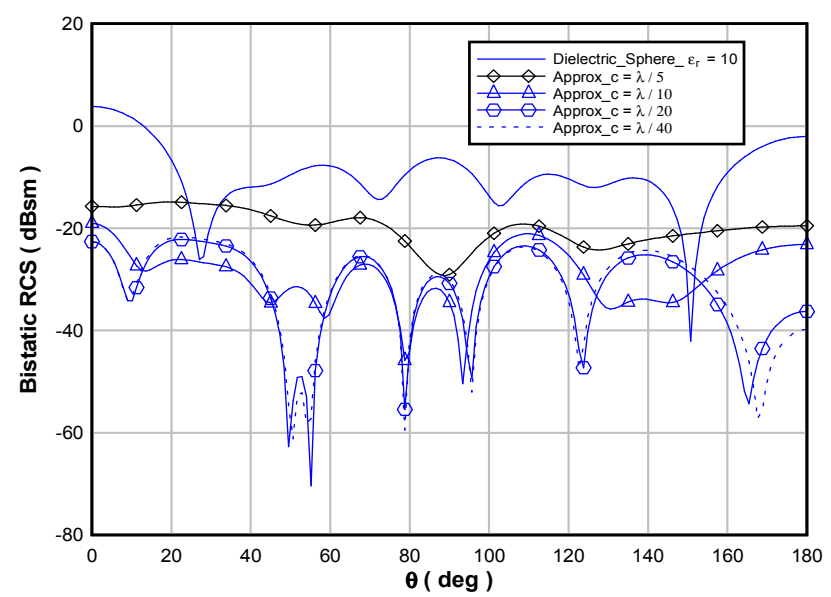

Fig. 27. Bistatic RCS for the concave-up nonlinear approximate cloaking for different values of the cloaking radii $c$ and $x=0.6$ for cloaked dielectric sphere with $\varepsilon_{d}=10$ (case III)

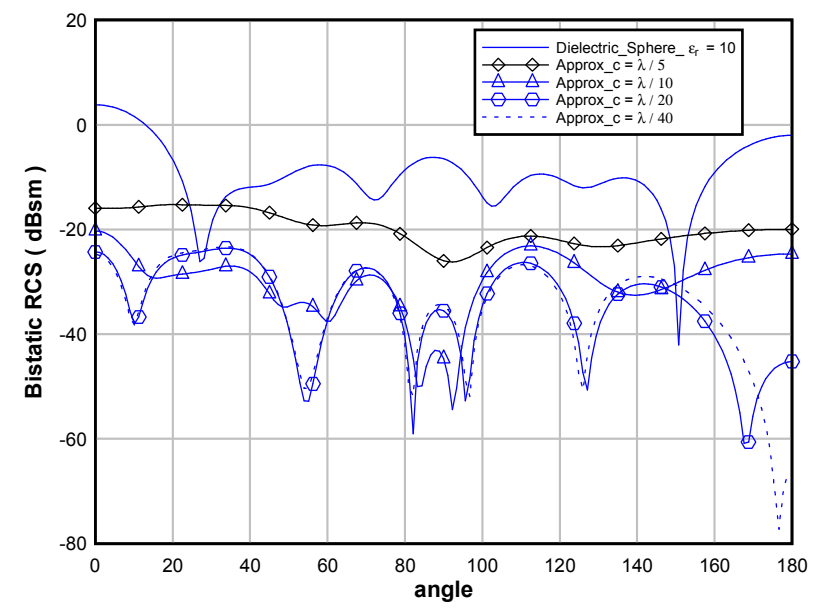

Fig. 28. Bistatic RCS for the concave-down nonlinear approximate cloaking for different values of the cloaking radii $c$ and $x=0.6$ for cloaked dielectric sphere with $\varepsilon_{d}=10$ (case III)

\subsubsection{Effect of the Nonlinearity Degree $x$}

Fig. 29 shows the effect of the nonlinearity degree $\mathrm{x}$ on the bistaic RCS of the concave-down nonlinear cloaks for cloaked dielectric sphere with $\varepsilon_{\mathrm{d}}=10$, for case II, $\mathrm{c}=$ $\mathrm{R}_{1} / 40$. The behavior is similar to the cloaked conducting sphere except for back scattering, Fig. 14. The results for case III are similar to those of Fig. 15 for the cloaked conducting sphere.

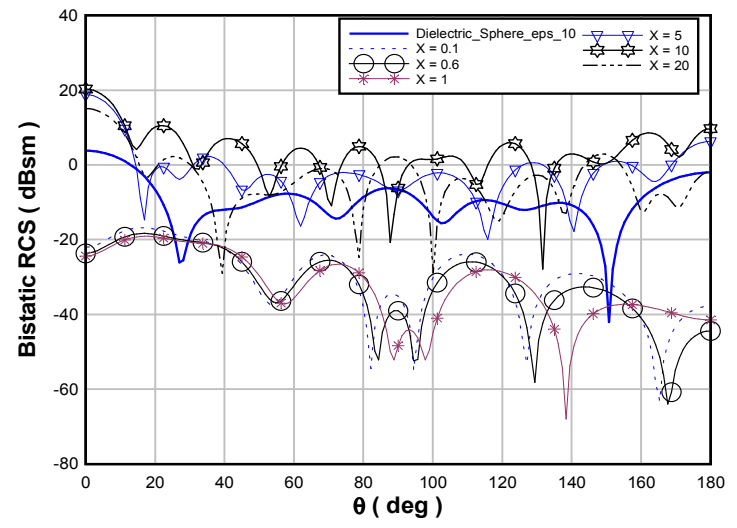

Fig. 29. Bistatic RCS for the concave-down nonlinear ideal cloaks for cloaked dielectric sphere with $\varepsilon_{d}=10$ for different values of $x$ (case II)

Fig. 30 shows the effect of the nonlinearity degree $\mathrm{x}$ on the bistaic RCS of the concave-up nonlinear cloaks for cloaked dielectric sphere with $\varepsilon_{\mathrm{d}}=10$, for case II. The results for case II are nearly identical to Fig. 16 for the conducting sphere. The concave-up results for case III are identical to those of the cloaked conducting sphere, Fig. 17.

From Figs. 29 and 30, it can be seen that for concave-down nonlinear transformations, the cloaking property is better retained when $\mathrm{x}<1$. As $\mathrm{x}$ increases $(x>1)$, their RCSs are increasing dramatically and can be larger than that of the uncloaked dielectric sphere (e.g., $x=5$, 10 and 20), which is not desired in the cloaking application.

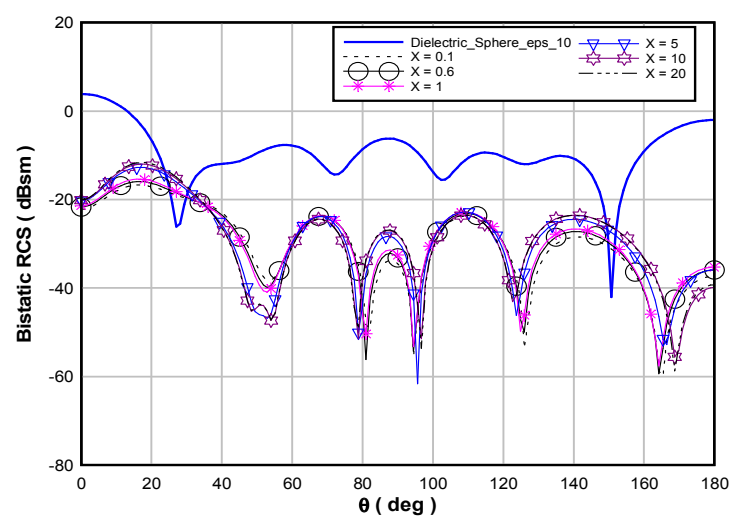

Fig. 30. Bistatic RCS for the concave-up nonlinear ideal cloaks for cloaked dielectric sphere with $\varepsilon_{d}=10$ for different values of $x$ (case II)

\subsubsection{Effect of Frequency and Reduced Radius $c$}

Figs. 31, 32 show the effect of the reduced radius $\mathrm{c}$ on the back scattering RCS of the concave-up and concave-down nonlinear cloaks, respectively, against the normalized frequency $R_{1} / \lambda$ for different values of $c, R_{1}=0.15 \mathrm{~m}$, $\mathrm{R}_{2}=2 \mathrm{R}_{1}$ for case II. 


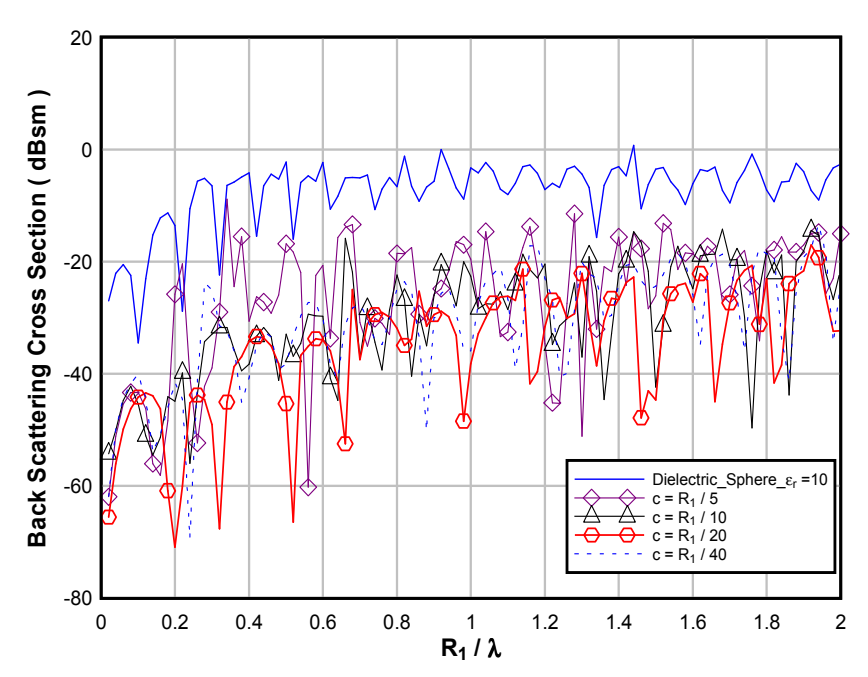

Fig. 31. The back scattering RCS for the concave-up nonlinear approximate cloaking for different values of the cloaking radii c and $x=0.6$ for cloaked dielectric sphere with $\varepsilon_{d}=10$ (case II)

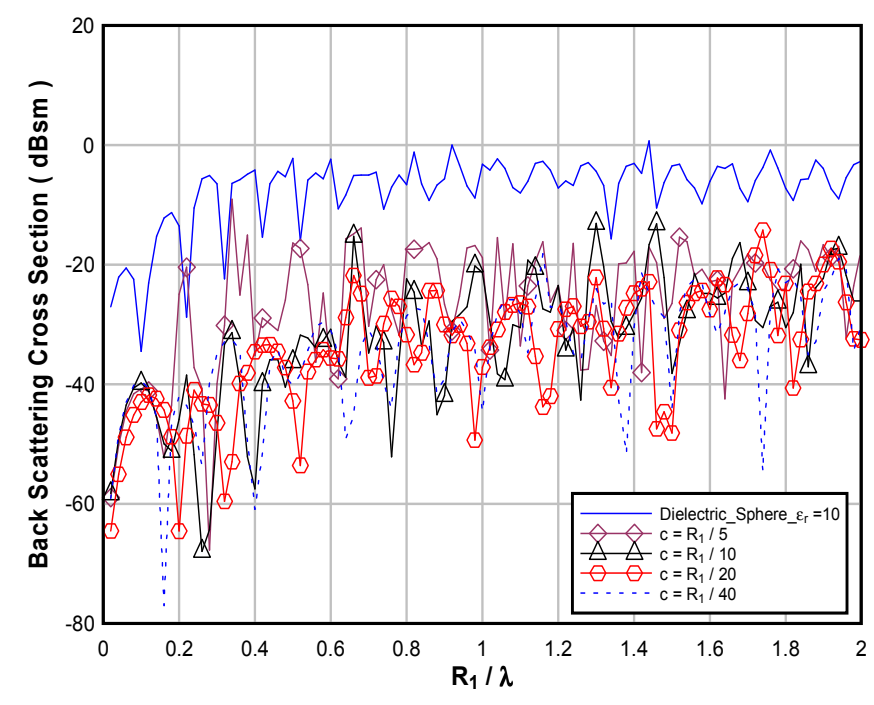

Fig. 32. The back scattering RCS for the concave-down nonlinear approximate cloaking for different values of the cloaking radii $c$ and $x=0.6$ for cloaked dielectric sphere with $\varepsilon_{d}=10$ (case II)

\subsubsection{Effect of Frequency and Nonlinearity Degree $x$}

The effect of the nonlinearity degree $\mathrm{x}$ on the back scattering properties versus normalized frequency $R_{1} / \lambda$ of an approximate cloaked dielectric sphere is investigated for the concave-down and concave-up nonlinear cloaks for both cases II and III with $\mathrm{c}=\mathrm{R}_{1} / 40$.

Fig. 33 and 34 show the back scattering RCS of the concave-up nonlinear approximate cloaking for cloaked dielectric sphere with $\varepsilon_{\mathrm{d}}=10$ for both cases II and III, respectively, for different values of the nonlinearity degree $\mathrm{x}$. The scattering properties have great similarities with those of the cloaked conducting sphere for case II, Fig. 22.

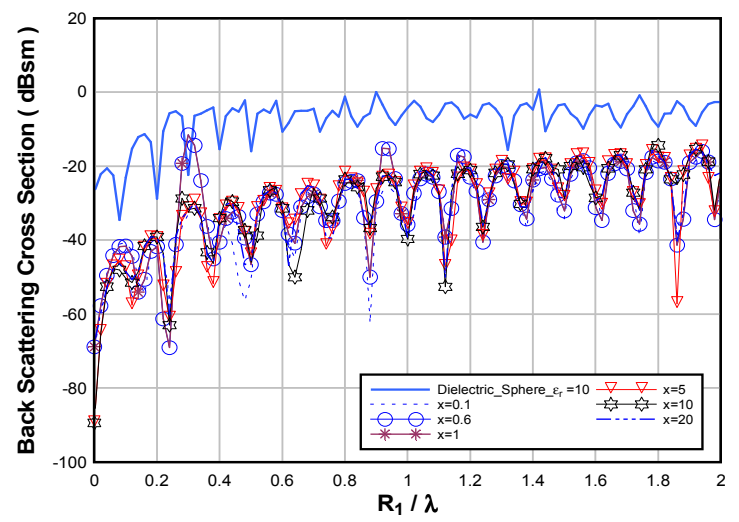

Fig. 33. The back scattering RCS for the concave-up nonlinear approximate cloaking for cloaked dielectric sphere with $\varepsilon_{d}=10$ for different values of $x$ (case II) at $c=R_{1} / 40$

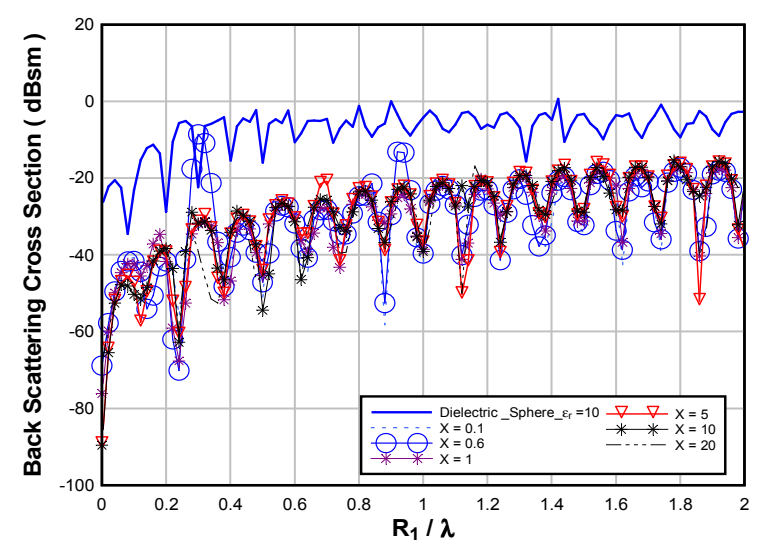

Fig. 34. The back scattering RCS for the concave-up nonlinear approximate cloaking for cloaked dielectric sphere with $\varepsilon_{d}=10$ for different values of $x$ (case III) at $c=R_{1} / 40$

Fig. 35 and 36 show the effect of the nonlinearity degree $x$ on the back scattering RCS for the concave-down nonlinear cloaks for cloaked dielectric sphere with $\varepsilon_{\mathrm{d}}=10$ for both cases II and III, respectively, at $c=\mathrm{R}_{1} / 40$. The scattering properties have great similarities with those of the cloaked conducting sphere for case II, Fig. 20. The scattering properties for case III have similarities with Fig. 21.

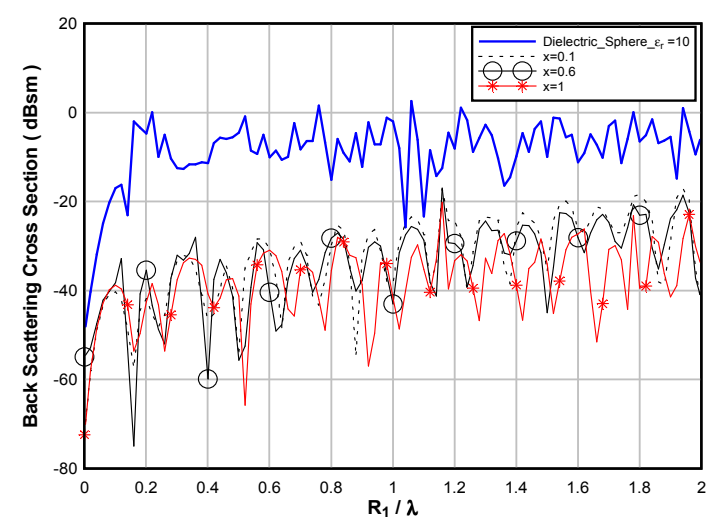

Fig. 35. The back scattering RCS for the concave-down nonlinear approximate cloaking for cloaked dielectric sphere with $\varepsilon_{d}=10$ for different values of $x$ (case II) at $c=R_{1} / 40$ 


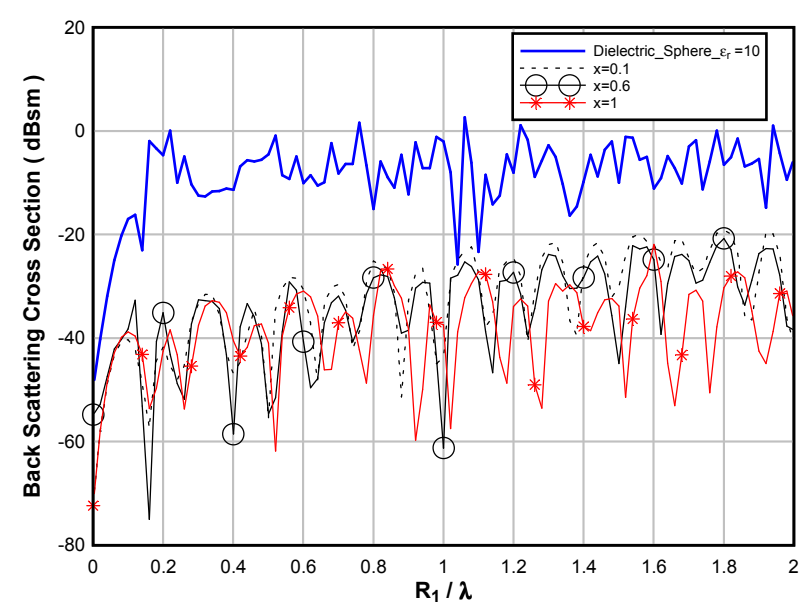

Fig.36. The back scattering RCS for the concave-down nonlinear approximate cloaking for cloaked dielectric sphere with $\varepsilon_{d}=10$ for different values of $x$ (case III) at $c=R_{1} / 40$

\subsection{Effect of Cloaking Material Thickness}

\subsubsection{Effect of Cloaking Material Thickness for Constant Layer Thicknesses}

Figs. 37, 38 show the effect of cloaking material thickness for constant layer thickness of $\lambda / 80$ on the bistatic RCS of the nonlinear approximate cloaking for cloaked conducting sphere for two different coating thicknesses of $\lambda / 2$ and $\lambda$ for concave-down and concave-up nonlinear cloaks, respectively. We use $c=R_{1} / 40$ and $x=0.6$. For fixed layer thickness, as the number of layers increases the average scattering decreases.

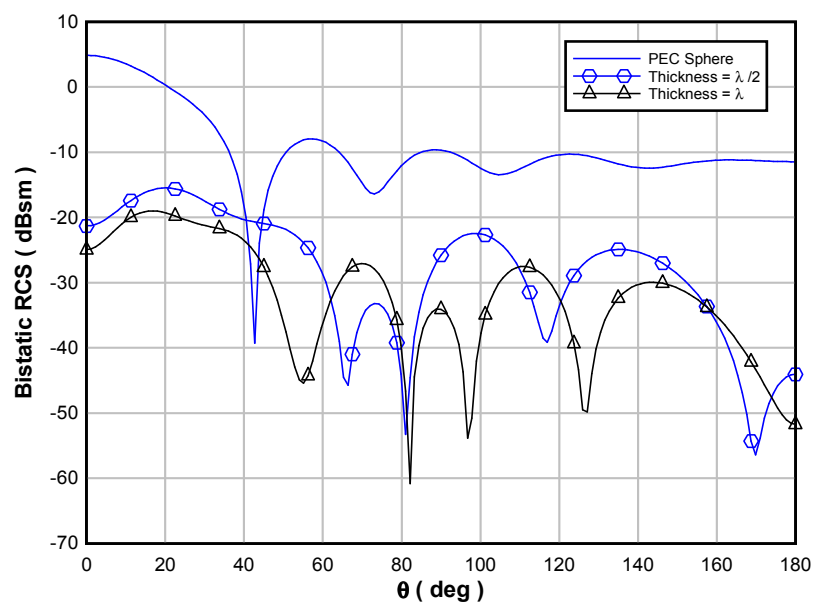

Fig. 37. Bistatic RCS for the concave-down nonlinear approximate cloaks for cloaked conducting sphere for two different thicknesses $\lambda / 2, \lambda$ and $c=R_{1} / 40, x=0.6$ (case II)

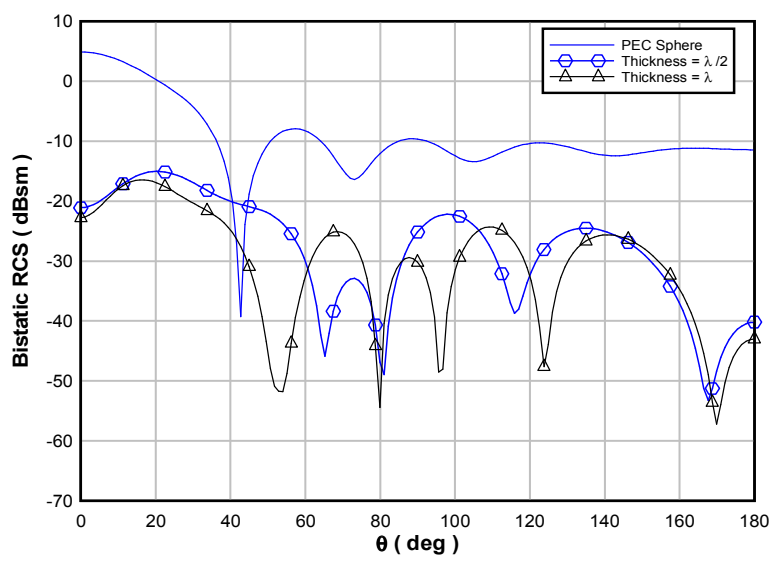

Fig. 38. Bistatic RCS of the concave-up nonlinear approximate cloaking for cloaked conducting sphere for two different thicknesses, $\lambda / 2, \lambda$ and $c=R_{1} / 40, x=0.6$ (case II)

\subsubsection{Effect of Cloaking Material Thickness for Constant Number of Layers}

Fig. 39 shows the effect of thickness of cloaking material on the bistatic RCS for the nonlinear approximate cloaking for cloaked conducting sphere for two different coating thicknesses of $\lambda / 2$ and $\lambda$ with 80 isotropic layers. Smaller cloaking thickness improves scattering since the normalized layer thickness decreases.

Fig. 40 shows the back scattering cross section versus normalized frequency $\mathrm{R}_{1} / \lambda$ of the concave-up nonlinear approximate cloaking for cloaked conducting sphere for two different thicknesses $\mathrm{R}_{2}=0.225 \mathrm{~m}, 0.3 \mathrm{~m}$, with $\mathrm{R}_{1}=0.15 \mathrm{~m}, \mathrm{c}=\mathrm{R}_{1} / 40$ and $\mathrm{x}=0.6$ ( case II ). On the average, smaller cloaking thickness improves scattering.

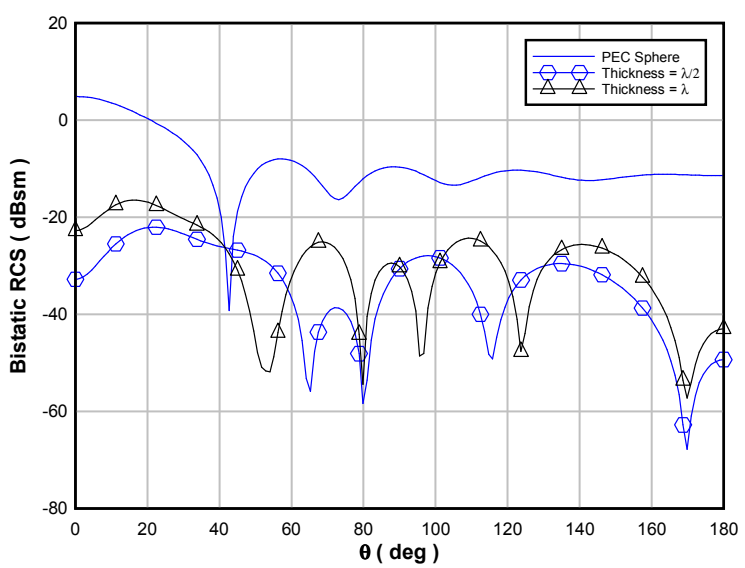

Fig. 39. Bistatic RCS for the concave-up nonlinear approximate cloaking for cloaked conducting sphere for two different thicknesses, $\lambda / 2, \lambda$ and $c=R_{1} / 40, x=0.6$ case $I I$ 


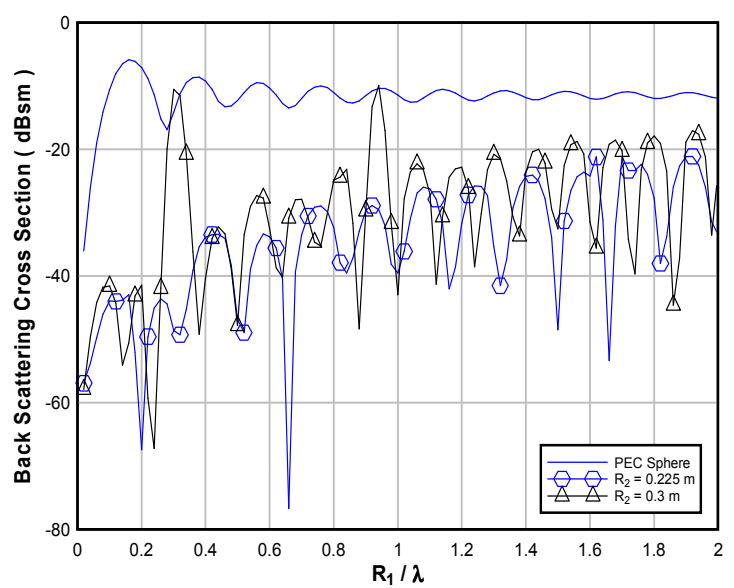

Fig. 40. The back scattering RCS for the concave-up nonlinear approximate cloaking for cloaked conducting sphere for two different thicknesses, $R_{1}=0.15 \mathrm{~m}, c=R_{1} / 40, x=0.6$ (case II)

\section{Conclusions}

The scattering properties of cloaked spherical bodies (conducting and dielectric) are studied using a combination of approximate cloaking, where the conducting sphere is transformed into a small sphere rather than to a point, and using two types of nonlinear transformations; concave-up and concave-down. The radially-dependent spherical cloaking shell is approximately discretized into many homogeneous anisotropic layers, provided that the thickness of each layer is much less than the wavelength, and this discretization raises the level of scattering as the number of layers decreases. Each anisotropic layer can be replaced by a pair of equivalent isotropic sub-layers, where the effective medium approximation is used to find the parameters of these two equivalent sub-layers.

This study is concerned with layering structure where a smaller value of $\mu$ is accompanied with the larger value of $\varepsilon$ in a layer, and the opposite combination in the second layer, which leads to large reduction of forward scattering with good reduction of back scattering. It is found that the reduced radius $\mathrm{c}$ has larger effect on the back scattering than on the forward scattering, and that good improvement on scattering is obtained for $c \leq \lambda / 20$. The use of reduced radius eliminates the zero value of the permittivity and the permeability at the radius of the body to be cloaked. Good reduction of scattering is achieved with nonlinearity exponentX $\leq 1$, and the concave-down profile given less back scattering.

The scattering performance from cloaked dielectric sphere is almost identical to that of a cloaked conducting sphere for $\mathrm{c}=0$, and in this case the scattering is actually a result of the interactions between the layers. Smaller thickness of the layers w.r.t. the wavelength results less scattering. The relative location of the layers with small and large values of $\varepsilon, \mu$ (cases II, III) has effect mainly on the scattering near the forward angles.

\section{References}

[1] J. B. Pendry, D. Schurig, and D. R. Smith, "Controlling electromagnetic fields," Science, Vol. 312, pp. 1780-1782, 2006.

[2] Q. Cheng, W. X. Jiang, and T. J. Cui, "Investigations of the electromagnetic properties of three-dimensional arbitrarily-shaped cloaks," Progress In Electromagnetics Research, Vol. 94, pp. 105 - 117, 2009.

[3] J. J. Yang, M. Huang, Y. L. Li, T. H. Li, and J. Sun, "Reciprocal invisible cloak with homogeneous metamaterials," Progress In Electromagnetics Research M, Vol. 21, pp. 105-115, 2011.

[4] A. Shahzad, F. Qasim, S. Ahmed, and Q. A. Naqvi, "Cylindrical invisibility cloak incorporating PEMC at perturbed void region," Progress In Electromagnetics Research M, Vol. 21, pp. 61-76, 2011.

[5] X. X. Cheng, H. S. Chen, and X. M. Zhang, "Cloaking a perfectly conducting sphere with rotationally uniaxial nihility media in monostatic radar system," Progress In Electromagnetics Research, Vol. 100, pp. 285-298, 2010.

[6] J. Zhang and N. A. Mortensen, "Ultrathin cylindrical cloak," Progress In Electromagnetics Research, Vol. 121, pp. 381-389, 2011.

[7] Y. B. Zhai and T. J. Cui, "Three-dimensional axisymmetric invisibility cloaks with arbitrary shapes in layered-medium background," Progress In Electromagnetics Research B, Vol. 27, pp. 151-163, 2011.

[8] D. Schurig, J. J. Mock, B. J. Justice, S. A. Cummer, J. B. Pendry, A. F. Starr, and D. R. Smith, "Metamaterial electromagnetic cloak at microwave frequencies," Science, Vol. 314, pp. 977-980, Oct. 2006.

[9] J.B. Pendry, A.J. Holden, D.J. Robbins, and W.J. Stewart, "Magnetism from conductors and enhanced nonlinear phenomena," IEEE Transactions on Microwave Theory and Techniques, Vol. 47, no. 11, pp. 2075 - 2084, Nov. 1999.

[10] G. V. Eleftheriades and K.G. Balmain, "Negative Refraction Metamaterials - Fundamental Principles and Applications," John Wiley, 2005.

[11] N. Engheta and R. W. Ziolkowski, "Metamaterials: Physics and Engineering Explorations," Wiley-IEEE Press, 2006.

[12] J. Wang, S. Qu, J. Zhang, H. Ma, Y. Yang, C. Gu, X. Wu, and $\mathrm{Z}$. $\mathrm{Xu}$, "A Tunable left-handed metamaterial based on modified broadside-coupled split-ring resonators," Progress In Electromagnetics Research Letters, Vol. 6, pp. 35-45, 2009.

[13] H. Liu, "Virtual reshaping and invisibility in obstacle scattering," Inverse Problems, Vol. 25, no. 4, pp. 1-10, 2009.

[14] T. Zhou, "Electromagnetic Inverse Problems and Cloaking," Ph.D. Thesis, Washington University, 2010.

[15] C. Qiu, L. Hu, B. Zhang, B. Wu, S. Johnsons and J. Joannopoulos, " Spherical cloaking using nonlinear transformations for improved segmentation into concentric isotropic coatings," Optics Express, Vol. 17, Iss. 16, pp. 13467-13478, 2009. 
[16] C. W. Qiu, L. Hu, and S. Zouhdi, "Isotropic non-ideal cloaks providing improved invisibility by adaptive segmentation and optimal refractive index profile from ordering isotropic materials," Optics Express, Vol. 18, no. 14, pp. 14950-14959, 2010.

[17] B. Zhang, "Study of Transformation-Based Invisibility Cloaks", Ph.D. Thesis, Massachusetts,2009.

[18] H. Chen, B. I. Wu, B. Zhang, and J. A. Kong, "Electromagnetic wave interactions with a metamaterial cloak,"Phys. Rev. Lett.99, pp. 14950-14959,2007.

[19] H. Zamel, E. El-Diwany and H. El-Hennawy, “Approximate electromagnetic cloaking of spherical bodies", 29th National Radio Science Conference (NRSC), Egypt, pp. 19-28, 2012.

[20] C. W. Qiu, L. Hu, X. Xu and Y. Feng, "Spherical cloaking with homogenous isotropic multilayered structures," Phys. Rev. E 79, pp. 047602 (1-4), 2009.

[21] C. Simovski and S. He, "Frequency range and explicit expressions for negative permittivity and permeability for an isotropic medium formed by a lattice of perfectly conducting $\Omega$ particles," Phys. Let. A, Vol. 311, Iss. 2-3, pp. 254-263, 2003.

[22] C. Simovski, and B. Sauviac, "Toward creating isotropic microwave composites with negative refraction," Radio Sci., Vol. 39, no. 2, Rs2014.1-18, 2004.

[23] C. M. Ji, P. Y. Mao and F. D. Ning, "An improved method of designing multilayered spherical cloak for electromagnetic invisibility," Chin. Phys. Let. , Vol. 27, no. 3, pp. 34102 (1-4), 2010 .

[24] M. Yan, W. Yan and M. Qiu, "Invisibility Cloaking by Coordinate Transformation," Progress in Optics, Vol. 52, pp. 261-304, 2009.

[25] J. A. Stratton, "Electromagnetic Theory," McGraw-Hill, 1941.

[26] R. F. Harrington, "Time Harmonic Electromagnetic Fields," McGraw-Hill, 1961.

[27] J. Jin, "Theory and Computation of Electromagnetic Fields," John Wiley, 2010.

[28] G. T. Ruck, D. E. Barrick, W. D. Stuart and C.K. Krichbaum, "Radar Cross Section Handbook," Kluwer Academic, 1970.

[29] O. Pena and U. Pal, "Scattering of electromagnetic radiation by a multilayered sphere," Computer Phy. Comm., Vol. 180, pp. 2348-2354, 2009.

[30] L. Kai and P. Massoli, "Scattering of electromagnetic plane waves by radially inhomogeneous spheres: a finely stratified sphere model," Applied Opt., Vol. 33, no. 3, pp. 501-511, Jan. 1994.

[31] A. Aden and M. Kerker, "Scattering of electromagnetic waves from two concentric spheres," Journal of Applied Physics, Vol. 22, no. 10, pp. 1242-1246, Oct. 1951. 